\title{
Elfn1-Induced Constitutive Activation of mGluR7 Determines Frequency-Dependent Recruitment of Somatostatin Interneurons
}

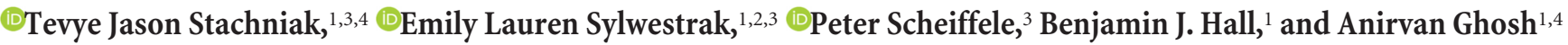 \\ ${ }^{1}$ F. Hoffmann-La Roche Ltd, Roche Innovation Center Basel, Basel 4051, Switzerland, ${ }^{2}$ Stanford University, Department of Bioengineering, Stanford, \\ California 94305, ${ }^{3}$ University of Basel, Departement Biozentrum, Basel 4056, Switzerland, and ${ }^{4}$ Biogen, Cambridge, Massachusetts 02142
}

Excitatory synapses onto somatostatin (SOM) interneurons show robust short-term facilitation. This hallmark feature of SOM interneurons arises from a low initial release probability that regulates the recruitment of interneurons in response to trains of action potentials. Previous work has shown that Elfn1 (extracellular leucine rich repeat and fibronectin Type III domain containing 1) is necessary to generate facilitating synapses onto SOM neurons by recruitment of two separate presynaptic components: mGluR7 (metabotropic glutamate receptor 7) and GluK2-KARs (kainate receptors containing glutamate receptor, ionotropic, kainate 2). Here, we identify how a transsynaptic interaction between Elfn1 and mGluR7 constitutively reduces initial release probability onto mouse cortical SOM neurons. Elfn1 produces glutamate-independent activation of mGluR7 via presynaptic clustering, resulting in a divergence from the canonical "autoreceptor" role of Type III mGluRs, and substantially altering synaptic pharmacology. This structurally induced determination of initial release probability is present at both layer $2 / 3$ and layer 5 synapses. In layer $2 / 3 \mathrm{SOM}$ neurons, synaptic facilitation in response to spike trains is also dependent on presynaptic GluK2-KARs. In contrast, layer 5 SOM neurons do not exhibit presynaptic GluK2-KAR activity at baseline and show reduced facilitation. GluK2-KAR engagement at synapses onto layer $5 \mathrm{SOM}$ neurons can be induced by calmodulin activation, suggesting that synaptic function can be dynamically regulated. Thus, synaptic facilitation onto SOM interneurons is mediated both by constitutive mGluR7 recruitment by Elfn1 and regulated GluK2-KAR recruitment, which determines the extent of interneuron recruitment in different cortical layers.

Key words: Elfn1; facilitation; GluK2; mGluR7; release probability; synapse

Significance Statement

This study identifies a novel mechanism for generating constitutive GPCR activity through a transsynaptic Elfn1/mGluR7 structural interaction. The resulting tonic suppression of synaptic release probability deviates from canonical autoreceptor function. Constitutive suppression delays the activation of somatostatin interneurons in circuits, necessitating high-frequency activity for somatostatin interneuron recruitment. Furthermore, variations in the synaptic proteome generate layer-specific differences in facilitation at pyr $\rightarrow$ SOM synapses. The presence of GluK2 kainate receptors in L2/3 enhances synaptic transmission during prolonged activity. Thus, layer-specific synaptic properties onto somatostatin interneurons are mediated by both constitutive mGluR7 recruitment and regulated GluK2 kainate receptor recruitment, revealing a mechanism that generates diversity in physiological responses of interneurons.

\section{Introduction}

Nervous system function is derived from both stable, genetically encoded neural circuit architecture, and dynamic, activity- dependent synaptic and cellular physiology. Synaptic proteins play a role in altering synaptic structure and regulating synaptic activity to generate neural circuit physiology. The leucine rich 
repeat (LRR)-containing superfamily of synaptic proteins conveys synaptic structure to pairs of connected neurons by regulating transsynaptic interactions with molecular specificity (de Wit and Ghosh, 2016). These synaptic proteins cannot only selectively generate connectivity between-specific cell types (Cao et al., 2015) or even subcellular regions (DeNardo et al., 2012), but can also determine the nature of the synaptic connection that is produced. Elfn1 (extracellular leucine-rich repeat fibronectin containing 1), a postsynaptic LRR-containing protein, is necessary to induce the electrophysiological properties of a synaptic connection between pyramidal neurons and somatostatin type interneurons (pyr $\rightarrow$ SOM) in the hippocampus (Sylwestrak and Ghosh, 2012). This strongly facilitating synapse regulates the recruitment of SOM interneurons by introducing a selective delay in spike generation as a result of the low initial release probability of incoming excitatory inputs, which in turn generates the distinct timing properties of these inhibitory interneurons (Pouille and Scanziani, 2004; Sylwestrak and Ghosh, 2012; Pala and Petersen, 2015). Elfn 1 alters the proteomic constitution of these synapses, as shown by the identification of mGluR7 as a presynaptic binding partner for Elfn1 (Tomioka et al., 2014), as well as the demonstration that Elfn 1 is necessary for the functional engagement of GluK2 subunit-containing kainate receptors (GluK2-KARs aka GluR6) at pyr $\rightarrow$ SOM synapses (Sylwestrak and Ghosh, 2012). The synaptic specificity of these receptors has long been recognized, along with the ability of these presynaptic components to change synaptic release properties (Shigemoto et al., 1996; Sun and Dobrunz, 2006; Pelkey and McBain, 2008; Sun et al., 2009; Tomioka et al., 2014). Elfn1 has been shown to play a structural role critical for synapse formation in retina (Cao et al., 2015). However, it is unclear whether Elfn 1 plays a passive structural role at pyr $\rightarrow$ SOM synapses by simply recruiting active presynaptic components, or whether Elfn 1 may actively alter receptor function to produce the characteristic physiological properties of pyr $\rightarrow$ SOM synapses. Target cell-specific synaptic release has also been described for cortical Layer 2/3 (L2/3) interneurons, both in cortical slices and in vivo (Reyes et al., 1998; Pala and Petersen, 2015). Here we examine how the target cellspecific proteomic substrates of a synapse may be regulated to give rise to layer-specific synaptic properties of pyr $\rightarrow$ SOM synapses.

\section{Materials and Methods}

Experimental model and subject details. All experimental protocols were conducted according to Swiss Laboratory Animal Science Association guidelines for animal research and were approved by the Roche Animal Care Committee and the Basel Cantonal Veterinary Committee.

Animals were housed on a $12 \mathrm{~h}$ light (06:00)/dark (18:00) cycle with ad libitum access to water and mouse chow. For characterizing synaptic responses, male and female mice (11- to 19-d-old) were used in cortical brain slice physiology experiments. Some off-target label in parvalbumin neurons has been reported (Hu et al., 2013) for the somatostatin-cre line used (Jackson ImmunoResearch Laboratories, Ssttm2.1(Cre)Zjh/J). Thus, following the initial characterization of wildtype (WT) versus knockout (KO) synaptic phenotypes, which included all cells, the subsequent pharmacological experiments were not conducted on cells that carried a stereotypical parvalbumin signature (depressing synapse) (Pouille and Scanziani, 2004; Tan et al., 2008). Elfn1 KO was from the Davis KOMP mouse repository (Elfn1tm1(KOMP)Vlcg), and the Ail4, lox-

T.J. Stachniak's present address: University of Zurich, Brain Research Institute, Winterthurerstrasse 190, Zurich, 8057 Switzerland

https://doi.org/10.1523/JNEUROSCI.2276-18.2019

Copyright $\odot 2019$ the authors
stop-lox-tdTomato was from Jackson ImmunoResearch Laboratories (B6.Cg-Gt(ROSA)26Sor < tm14(CAG-tdTomato)Hze/J).

Electrophysiology. Coronal brain slices from sensory cortex (bregma $\sim-0.5$ to $-2.0 \mathrm{~mm}$ ) from SOM-cre $\times$ tdTom or Elfn1 KO $\times$ SOMcre $\times$ tdTom mice were prepared in cold cutting solution containing the following (in $\mathrm{mm}$ ): 75 sucrose, $87 \mathrm{NaCl}, 25 \mathrm{NaHCO}_{3}, 25 \mathrm{D}$-glucose, 2.5 $\mathrm{KCl}, 7 \mathrm{MgCl}_{2}$, and $1.25 \mathrm{NaH}_{2} \mathrm{PO}_{4}$, aerated with $95 \% \mathrm{O}_{2} / 5 \% \mathrm{CO}_{2}$. Slices were transferred to aCSF containing the following (in $\mathrm{mM}$ ): $120 \mathrm{NaCl}, 26$ $\mathrm{NaHCO}_{3}, 10$ D-glucose, $3 \mathrm{KCl}, 2 \mathrm{MgCl}_{2}, 2 \mathrm{CaCl}_{2}$ and $1.25 \mathrm{NaH}_{2} \mathrm{PO}_{4}$, aerated with $95 \% \mathrm{O}_{2} / 5 \% \mathrm{CO}_{2}$. The intracellular solution contained the following (in $\mathrm{mm}$ ): $125 \mathrm{~K}$ gluconate, $2 \mathrm{KCl}, 10 \mathrm{HEPES}, 10$ phosphocreatine, $4 \mathrm{MgCl}_{2}$, 1 EGTA, 0.1 $\mathrm{CaCl}_{2}$, 4 ATP, $0.4 \mathrm{GTP}$, pH 7.35, 290 mOsm.

Human embryonic kidney (HEK) cell recordings were in HEPES buffered saline containing the following (in $\mathrm{mm}$ ): $135 \mathrm{NaCl}, 5 \mathrm{HEPES}, 10$ D-glucose, $5 \mathrm{KCl}, 1.2 \mathrm{MgCl}_{2}$, and 2.6 $\mathrm{CaCl}_{2}, \mathrm{pH} 7.2-7.4$.

Bath-applied compounds NS102 (20 mM, Sigma-Aldrich, N179, CAS: 136623-01-3) and AMN082 (1 mM, VWR, Ab120011, CAS: 97075-46-2) were dissolved as stock solutions in DMSO; methylserine-O-phosphate (MSOP, 50 mm, Tocris Bioscience, 0803, CAS: 66515-29-5) and L-AP4 (Enzo, ALX-550-026; CAS: 23052-81-5, pH 7.4) were dissolved in water. HEK cell recordings were done in HEPES buffered saline, and Elfn1-Fc $(\sim 10 \mu \mathrm{M})$ and MAb $1 / 28(2.5 \mathrm{mg} / \mathrm{ml}, \sim 20 \mu \mathrm{M})$ were applied directly to cells $(2.5 \mu \mathrm{l}$ into $\sim 2.5 \mathrm{ml}$ for $\sim 1000 \times$ dilution $)$.

Cell culture. HEK 293 cells were grown to 90\%-95\% confluency in 6 well plates (with DMEM, 10\% horse serum, penicillin/streptomycin), and transfected with $2 \mu \mathrm{g}$ DNA/well using Lipofectamine 2000 (Invitrogen). Cells were washed, collected with trypsin/EDTA, pelleted, resuspended in $1 \mathrm{ml}$ DMEM, and replated at a 1:3 dilution onto poly-1lysine-coated coverslips. Coplated cells were incubated $15-48 \mathrm{~h}$, then fixed in 4\% paraformaldehyde (PFA) for $30 \mathrm{~min}$, stained with anti-ZO1 (mouse 1:100 Invitrogen \#339100; secondary goat anti-mouse 647), and mounted onto slides with Prolong Gold medium. Surface fluorescence at cell-cell contact sites was quantified at ZO1-labeled tight junctions and was normalized within the same cell to surface fluorescence at junctions contacting unlabeled cells. Fluorometric imaging plate reader (FLIPR) assay was performed with the FLIPR Calcium 6 Assay on a Hamamatsu FDSS 7000 using a mGluR7 HEK cell line provided by L. Lindemann (Lindemann et al., 2011). For FLIPR, Elfn-Fc concentration was diluted $100 \times$.

Elfn1-Fc DNA $(10 \mu \mathrm{g})$ was transfected into HEK 293 cells plated at $80 \%$ confluency on T75 culture flasks with Lipofectamine 2000. Extracellular culture medium was collected at $48-72 \mathrm{~h}$ and run through a NAb protein A spin column. The column eluate was adjusted to $\mathrm{pH} 7$ and then concentrated in Amicon Centrifugal $4 \mathrm{ml} 30 \mathrm{kDa}$ filters to generate concentrated Elfn 1 Fc protein.

Primary cortical neurons were cultured from C57BL/6 E16.5 mouse embryos. Neurons were maintained in Neurobasal-A medium, supplemented with B27, glucose, glutamax, and penicillin/streptomycin at a cell density of $15,000 /$ well in 96 well plates $\left(441 \mathrm{cells} / \mathrm{mm}^{2}\right)$. Cells were fixed on DIV 14 or DIV 20 with 4\% PFA for $60 \mathrm{~min}$.

Histochemistry. Immunostaining of $50 \mu \mathrm{m}$ coronal brain sections was as follows: following transcardial perfusion with $4 \%$ PFA and overnight after fixation, $1 \mathrm{~h}$ blocking in normal donkey serum with $0.2 \%$ Triton $\mathrm{X}-100$ was followed by overnight incubation in primary antibody, followed by $2 \mathrm{~h}$ in secondary antibody. Primary antibodies were as follows: mouse monoclonal anti-ZO-1 (Invitrogen, ZO1-1A12), rabbit anti-Elfn (anti-lrrc62, Prestige Antibodies, Sigma-Aldrich, HPA000781), rabbit anti-GluR6/7 (anti-GluK2/3, Millipore, 04-921), anti-mGluR7 rabbit polyclonal antibody (Millipore, 07-239), anti-mGluR7 mouse monoclonal antibody (MAB1/28, custom Roche Diagnostics antibody) (Ullmer et al., 2012), control antibody (donkey anti-chicken 488, Jackson ImmunoResearch Laboratories, 703-545-155), and rat anti-somatostatin (Santa Cruz Biotechnology, SC-7819).

ISH for GRIK2 mRNA and Elfn1 mRNA was performed using the protocol from the RNAScope multiplex ISH kit (ACDBio, 320850).

Western blot. Dissection of hippocampus from 6-week-old mice was followed by tissue homogenization in lysis buffer (containing $50 \mathrm{~mm}$ Tris, pH 7.5, 150 mm NaCl, 5 mm EDTA, 1\% Triton X-100, 10\% glycerol, 1 mm PMSF; Roche Diagnostics protease inhibitors), with $20 \mathrm{~s}$ shaking using 
Precellys ceramic bead system. MAB1/28 immunoprecipitation of mGluR7 was done from $500 \mu \mathrm{l}$ of cortical lysates ( $\sim 100 \mathrm{mg}$ tissue), incubated overnight in a NAb protein A spin column prebound $(1 \mathrm{~h})$ to $1 \mu \mathrm{g}$ of MAB1/28. The column was washed twice with lysis buffer, once with phosphate buffered saline (PBS), then eluted with $500 \mu \mathrm{l} 2 \times$ SDS buffer. Sample eluate $(20 \mu \mathrm{l})$ was run beside sample input $(2 \mu \mathrm{l})$ with reducing agent on a $4 \%-12 \%$ Bis-Tris gel in 3-(N-morpholino) propanesulfonic acid buffer for $2.5 \mathrm{~h}$. Gels were blotted overnight onto PVDF membranes in transfer buffer with $0 \%$ methanol. Incubation overnight with primary then $2 \mathrm{~h}$ in anti-rabbit IR800 secondary was followed by exposure on Licor Odyssey IR scanner (Li-Cor). Representative blot was verified with at least two biological replicates and at least three technical replicates.

Coimmunoprecipitation of mGluR7 was done from $500 \mu$ l of cortical lysates ( $\sim 50 \mathrm{mg}$ tissue) in lysis buffer using a Dounce homogenizer. GluK2-GFP/mGluR7-GFP-expressing HEK cells were collected with trypsin/EDTA, pelleted, washed in cold PBS, and lysed in lysis buffer. Lysates were spun at $100,000 \times g$ for $1 \mathrm{~h}$ at $4^{\circ} \mathrm{C}$, then stored at $-20^{\circ} \mathrm{C}$. Samples incubated overnight with $2.5 \mu \mathrm{g}$ of MAB1/28 or $1 \mu \mathrm{g}$ of rabbit anti mGlur7 and spun at 140,00 rpm for $10 \mathrm{~min}$. Protein A Sepharose beads $(30 \mu \mathrm{l})$ were added, with end-over-end mixing at $4^{\circ} \mathrm{C}(4 \mathrm{~h})$. Beads were pelleted at $3000 \mathrm{rpm}$ for $2 \mathrm{~min}$, the unbound fraction saved for analysis, and then washed $3 \times$ with $500 \mu$ l lysis buffer, $1 \times$ with PBS, then pelleted at 14,000 rpm for $1 \mathrm{~min}$. Beads were resuspended in $30 \mu \mathrm{l}$ of $2 \times$ SDS buffer (200 mм DTT, 100 mм Tris-HCl, pH 6.8, 20\% glycerol, $4 \%$ SDS, bromophenol blue $0.025 \%$ ), boiled at $95^{\circ} \mathrm{C}$ for $5 \mathrm{~min}$, spun down at $14,000 \mathrm{rpm}$ for $5 \mathrm{~min}$, then analyzed by Western blot.

Elfn1-tdTomato-expressing HEK cells were collected with trypsin/ EDTA, pelleted, and washed in cold PBS. Crosslinking was performed with bis[sulfosuccinimidyl]suberate (BS3), applied either to cell suspensions at $0.1-3 \mathrm{~mm}$ in $500 \mu \mathrm{l}$ PBS, or to $0.5 \mu \mathrm{g}$ purified Elfn-Fc protein at $3-100 \mu \mathrm{M}$ in $20 \mu \mathrm{l} \mathrm{PBS}$ for $30 \mathrm{~min}$ at room temperature, quenched with $20 \mu \mathrm{l} 0.5 \mathrm{~m}$ Tris- $\mathrm{HCl}, \mathrm{pH}$ 6.8, for $15 \mathrm{~min}$. Cells were lysed in $50 \mathrm{~mm}$ Tris, pH 7.5, $150 \mathrm{~mm} \mathrm{NaCl}, 5$ mm EDTA, 1\% Triton X-100, 0.1\% SDS, $0.1 \mathrm{~mm}$ beta mercaptoethanol; protease inhibitors and were incubated on ice for $1 \mathrm{~h}$, spun at $14,000 \mathrm{rpm}$ for $10 \mathrm{~min}$, then stored at $-20^{\circ} \mathrm{C}$. Western blotting was done on a $4 \%-20 \%$ mini-PROTEAN TGX gel (Bio-Rad, 456-1093) gel, run in SDS buffer. Protein was transferred to a nitrocellulose membrane in $10 \%$ propranolol in SDS buffer, blocking in TBS Tween $1 \%$ with $5 \%$ milk powder. Incubation overnight with primary then $2 \mathrm{~h}$ in HRP secondary was followed by exposure using Advansta WesternBright Sirius detection reagent (K-12043-D20) on a ChemiDoc MP imaging system (Bio-Rad). Representative blots were verified with at least two biological replicates and at least three technical replicates.

Image acquisition and analysis. Images were captured on the Operetta High-Content Imaging System (PerkinElmer) with $20 \times$ WD objective in nonconfocal mode, an SP5 confocal microscope (Leica Microsystems) or an Olympus FV1000 confocal microscope. $Z$ stacks were collapsed in a maximum projection and analyzed using LAS software (Leica Microsystems) or ImageJ.

Plasmids. The Elfn1-tdTomato plasmid was created by swapping tdTomato for the green fluorescent protein (GFP) label in Efln1-GFP (Sylwestrak and Ghosh, 2012), containing the pEGFPN1 backbone. Elfn1-Fc plasmid was created by swapping Elfn 1 ectodomain for the CASPR ectodomain in 3CPro backbone from Davide Comoletti (Rubio-Marrero et al., 2016). The $\Delta$ LRR-Elfn1-GFP plasmid was created by subcloning $\Delta$ LRR-Elfn1 into pEGFPN1 backbone. The $\Delta$ ecto-mGluR7-GFP plasmid was created by custom synthesis of $\Delta$ ecto-mGluR7 and subcloning into pCMV6-AC-GFP. mGluR7-GFP and GRIK2-GFP plasmids were purchased from Origene (RG217402, RG222369).

Experimental design and statistical analysis. Potassium reversal potential for HEK cell recordings was $-82 \mathrm{mV}$. The holding potential for neuronal voltage-clamp recordings was $-70 \mathrm{mV}\left(\mathrm{Cl}^{-} \mathrm{E}_{\text {rev }}\right)$. Hippocampal recordings used a bipolar stimulating electrode positioned in the alveus. Cortical recordings used a monopolar stimulation through a glass pipette positioned in Layer 2/3 (L2/3) or Layer 5 (L5) of sensory cortex in acute slices from SOM-cre $\times$ tdTomato (Ai14) mice. The stimulating electrode was placed typically $50-150 \mu \mathrm{m}$ from the recorded cell, moving parallel to the pial surface. Stimulation within a cortical layer was thus presumed to preferentially recruit activity from local neurons. However, the extensive nature of cortical connectivity makes it impossible to rule out the possibility of excitation of synaptic inputs from distal sites. Representative traces are an average of 12 sweeps ( 2 min of data).

For synaptically induced SOM firing, action potentials were recorded with loose-seal patch in response to synaptic stimulation $(10 \times 50 \mathrm{~Hz}$ stim, 50-100 $\mu \mathrm{s}, 30-100 \mu \mathrm{A}$, set $+10 \mu \mathrm{A}$ above synaptic spike threshold). Stimulation intensities were as follows: L2/3 WT, $50 \pm 5 \mu \mathrm{A}, \mathrm{L} 2 / 3$ KO $49 \pm 3 \mu \mathrm{A}$, L5 WT, $52 \pm 3 \mu \mathrm{A}$. Time to half-maximum spiking was calculated by fitting the average spike probability from 10 sweeps of $10 \times$ $50 \mathrm{~Hz}$ stimuli using the MATLAB dose_response script (Evangelista, 2004). For Elfn $1 \mathrm{KO}$, the first 5 of the $10 \times 50 \mathrm{~Hz}$ events were fit, as these made better fits than when including the late events where the maximum spiking probabilities were not maintained. Plots of spike probability show the average of the first 5 of 10 events, for clarity.

Quantal amplitude in WT and Elfnl KO was calculated by fitting spontaneous excitatory postsynaptic current (EPSC) amplitude distribution histograms with the multiple Gaussian curve fitting function in Clampfit.

Synaptic release probability was calculated using a similar method to the variance-mean analysis described by Oleskevich et al. (2000). Briefly, the variance from each of $5 \times 50 \mathrm{~Hz}$ synaptic currents from 10 or more consecutive sweeps was plotted as a function of mean amplitude. The rapidly changing release probability for synaptic currents $1-5$ resulted in a curve that for most cells could be fit with the following equation:

$$
y=A x-B x^{2}
$$

$y$ is the variance, $x$ is the mean amplitude, and coefficients $A$ and $B$ were then used to calculate release probability for a given amplitude " $x$ " as follows:

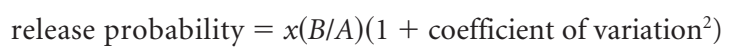

The coefficient of variation (SD/mean) was estimated to be 0.5 on average, based on asynchronous release events sampled from a subset of the data. For cells that could not be fit with $y=A x-B x^{2}$ with an $r^{2}>0.3$, the release probability calculation was excluded. The number of excluded cells for L2/3 WT was $1 / 24$, for L2/3 KO was $2 / 24$ for L5 WT was $4 / 24$, for L5 $\mathrm{KO}$ was $8 / 24$.

Data are mean \pm SEM. Number of measurements $n / N$ represents $n$ cells recorded from $N$ animals, typically using 1 cell per slice. For HEK cell experiments $n / N$ represents $n$ cells from $N$ biological replicates. Statistical testing was done in MATLAB. Comparisons within conditions were made by two-tailed paired Student's $t$ test, treatment versus baseline. Comparisons across conditions or between genotypes were done with an unpaired $t$ test assuming unequal variance. For multiple comparisons, a one-way or two-way ANOVA was done with a Bonferroni post hoc test. Holm's correction for multiple comparisons has been applied to $p$ values (listed as "corrected $p$ ") wherever L2/3 WT data were compared first to $\mathrm{L} 2 / 3 \mathrm{KO}$ data and then later to L5 data.

\section{Results}

\section{Interneurons in Elfn1 KO mice show reduced synaptic facilitation}

We had previously reported that shRNA-mediated knockdown of Elfn1 in oriens-lacunosum molecular (OLM) cells (SOM interneurons) in hippocampus leads to a marked reduction in synaptic facilitation of pyr $\rightarrow$ SOM synapses, whereas introduction of Elfn 1 into parvalbumin interneurons is sufficient for the cellautonomous induction of facilitation at those synapses (Sylwestrak and Ghosh, 2012). Synaptic facilitation arising from high-frequency stimulation results in the preferential recruitment of dendritic targeting OLM cells rather than soma targeting interneurons (Pouille and Scanziani, 2004). To determine whether cortical SOM neurons are likewise recruited by highfrequency stimulation, we examined synaptic facilitation at pyr $\rightarrow$ SOM synapses in L2/3 of sensory cortex in acute slices from 

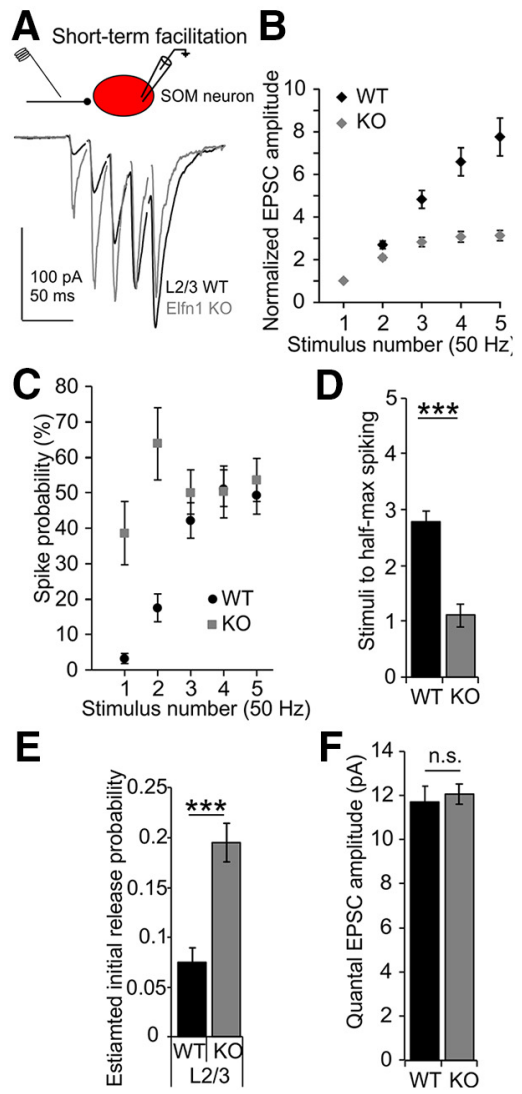

Figure 1. Elfn1 generates facilitating pyr $\rightarrow$ SOM synapses in cortex. $A$, Recording from layer 2/3 SOM neurons, high-frequency stimulation results in substantial facilitation of excitatory transmission, which is reduced in Elfn $1 \mathrm{KO} . \boldsymbol{B}, \mathrm{In} \mathrm{L} 2 / 3$ of cortex, Elfn $1 \mathrm{KO}$ has a reduced synaptic facilitation ratio at $50 \mathrm{~Hz}$. ESPC amplitude is normalized to first EPSC amplitude. $\boldsymbol{C}$, Within a $50 \mathrm{~Hz}$ train of synaptic-driven $\mathrm{SOM}$ activation, spiking onset is delayed in L2/3 neurons. Spiking onset is rapid in L2/3 Elfn1 KO. D, Probability of spiking takes longer to reach halfmaximum in WT compared with $K 0$ neurons. $E$, Initial release probabilities calculated for $L 2 / 3$ differ between WT and KO. F, Quantal EPSC amplitude does not differ from WT to KO. Data are mean \pm SEM. ${ }^{* * *} p<0.001$. n.S., Not significant $(p>0.05)$.

SOM-cre $\times$ tdTomato (Ai14) mice. Labeled neurons in L2/3 showed a strong short-term facilitation of excitatory transmission in response to high-frequency stimulation $(50 \mathrm{~Hz}$; Fig. 1A) that occurred in conjunction with a delay of synaptic induced spiking, as seen previously for hippocampal SOM interneurons (Pouille and Scanziani, 2004; Sylwestrak and Ghosh, 2012). Genetic deletion of Elfn1 reduced the facilitation ratio (EPSC5/ EPSC1 ratio: $3.1 \pm 0.2$ in $\mathrm{KO}$ vs $7.7 \pm 0.9$ in WT, corrected $p=$ $0.00006, t=5.02, t$ test, $n / N=24 / 6$ and $24 / 9$, respectively, $5 \times 50$ $\mathrm{Hz}$; Fig. $1 B$ ) and the recruitment delay for L2/3 SOM neurons compared with WT (stimuli to half-maximum spiking: $1.1 \pm 0.3$ in KO, vs $2.8 \pm 0.2$ in WT, corrected $p=1 \times 10^{-6}, t=6.05$, $t$ test, $n / N=16 / 2$ and $24 / 4$, respectively, $5 \times 50 \mathrm{~Hz}$; Fig. $1 C, D$ ). L2/3 pyr $\rightarrow$ SOM synapses showed an increased initial release probability in Elfn1 KO animals (Fig. 1E; calculated from mean-variance analysis) (Oleskevich et al., 2000). Conversely, Elfn1 KO produced no change in quantal amplitude, as calculated from spontaneous EPSC amplitude distributions (quantal amplitude: $12.1 \pm 0.5$ in $\mathrm{KO}$ vs $11.7 \pm 0.7$ in WT, $p=0.7, t=0.42, t$ test, $n / N=6 / 1$ and $8 / 3$, respectively; Fig. $1 F$ ). As shown previously for miniature EPSC amplitudes during shRNA knockdown of Elfn1 (Sylwestrak and Ghosh, 2012), the similar synaptic amplitudes in WT and KO indicate that Elfn1 removal does not affect postsynaptic properties. Thus, a prominent feature of pyr $\rightarrow$ SOM syn- aptic facilitation and delay-to-spiking is the low initial release probability.

\section{Elfn1 recruitment of mGluR7 constitutively reduces synaptic release probability}

Elfn1 KO mice exhibit hyperactivity and handling induced seizures (Dolan and Mitchell, 2013; Tomioka et al., 2014). A similar phenotype is observed in mGluR7 KO mice (Sansig et al., 2001), which predicted the subsequent identification of mGluR7 as a presynaptic binding partner of Elfn1 (Tomioka et al., 2014). Elfn1 KO substantially reduces mGluR7 immunoreactivity at synapses onto somatostatin neurons in hippocampus (Tomioka et al., 2014). Constitutive activity of mGluRs is thought to contribute to the properties of this synapse (Losonczy et al., 2003), and mGluR7 homodimerization has been proposed as a mechanism by which such constitutive activity may be generated (Kammermeier, 2015; Levitz et al., 2016). We therefore hypothesized that Elfn1 may influence release probability through promoting constitutive activity of mGluR7. In cortical cultures, mGluR7 is selectively clustered along the dendritic arbor of SOM neurons where it colocalizes with Elfn1 at synapses (Fig. 2A). In brain slices, mGluR7 dendritic clustering is also visible on SOM interneurons in L2/3 of cortex (Fig. 2B), and is disrupted by Elfn 1 deletion (Fig. 2C). Elfn1 coimmunoprecipitates mGluR7, as well as other Type III mGluRs, indicating that these proteins physically interact (Tomioka et al., 2014; Dunn et al., 2018). We assessed the effects of mGluR7 on synaptic transmission at pyr $\rightarrow$ SOM synapses by antagonizing mGluR7 with the mGluR (Type III)-selective antagonist MSOP (100 $\mu \mathrm{M})$. MSOP increased synaptic transmission at pyr $\rightarrow$ SOM synapses in L2/3 (EPSC amplitude: $63 \pm 10 \mathrm{pA}$ in MSOP vs $55 \pm 10 \mathrm{pA}$ baseline, $p=0.002, t=$ 3.82 , paired $t$ test, $n / N=14 / 8$; Fig. $2 D)$. Surprisingly, the change in synaptic transmission was primarily restricted to a change in the amplitude of the first EPSC. The effects of MSOP were reversible, with washout of compound restoring first EPSC amplitude to baseline. The MSOP-mediated increase in initial release was absent in Elfn1 $\mathrm{KO}$ animals (EPSC amplitude, L2/3 KO: $150 \pm 49$ $\mathrm{pA}$ in MSOP vs $160 \pm 54 \mathrm{pA}$ at baseline, $p=0.3, t=1.25$, paired $t$ test, $n / N=6 / 2$; Fig. $2 E$ ). The ability of the mGluR7 antagonist MSOP to increase initial release probability in L2/3 suggests a novel mechanism of action for Elfn1/mGluR7 signaling. Contrary to the expectation that mGluR7 is responding to synaptic glutamate, the constitutive reduction in synaptic release probability is consistent with an agonist independent constitutive mGluR7 activity and is not found in Elfn1 KO (Fig. 2F). The loss of constitutive activity in Elfn1 $\mathrm{KO}$ was not due to a reduction in total mGluR7 protein levels in cortex (Fig. $2 G$ ), but rather due to a redistribution mGluR7 protein localization away from the discrete dendritic clustering that is visible in WT (Fig. $2 H, I$ ). These results are consistent with previous findings in the hippocampus (Tomioka et al., 2014).

To further characterize this novel mode of action for synaptic mGluR7, we activated mGluR7 directly with the Type III mGluR agonist L-AP4. L-AP4 caused no further suppression of initial release in WT slices but suppressed initial release in L2/3 in Elfn1 KO slices (EPSC amplitude, L2/3 WT: $16 \pm 3$ pA in $30 \mu \mathrm{M} \mathrm{L}-\mathrm{AP} 4$ vs $18 \pm 3 \mathrm{pA}$ at baseline, $p=0.3, t=1.06$, paired $t$ test, $n / \mathrm{N}=$ 11/5, L2/3 KO: $54 \pm 12$ in $30 \mu \mathrm{M} \mathrm{L}-\mathrm{AP} 4$ vs $77 \pm 16$ at baseline, $p=$ $0.007, t=4.86$, paired $t$ test, $n / N=9 / 2$; Fig. $2 J$ ). The lack of suppression in WT slices was not likely due to a "floor effect" or to a reduction in total receptor expression levels, as high-dose L-AP4 $(300 \mu \mathrm{M})$ still produced strong suppression of initial release in both Elfn $1 \mathrm{WT}$ and KO slices (EPSC amplitude, L2/3 WT: $28 \pm 8$ 


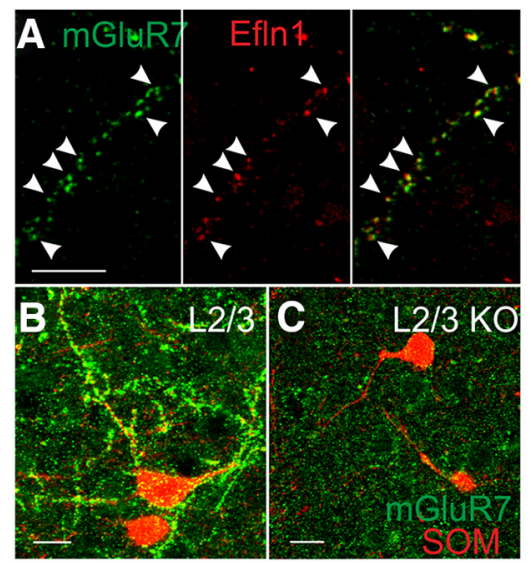

D mGluR7 blockade in WT L2/3
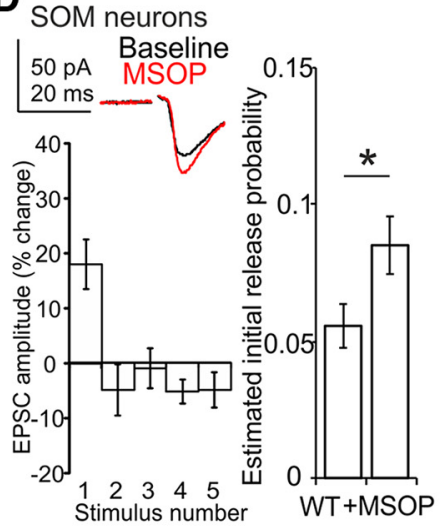

E mGluR7 blockade in KO L2/3

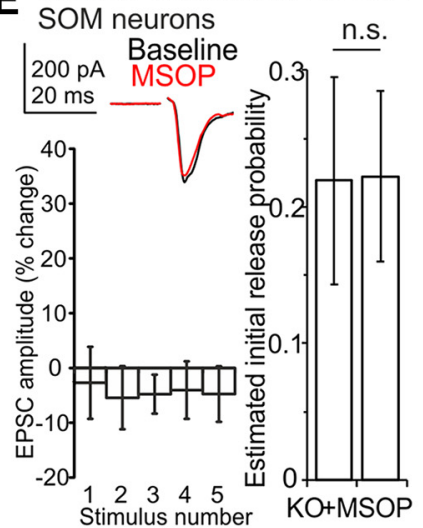

FElfn1 WT SOM synapses Elfn1 WT SOM synapses Elfn1 WT SOM synapses Elfn1 KO SOM synapses
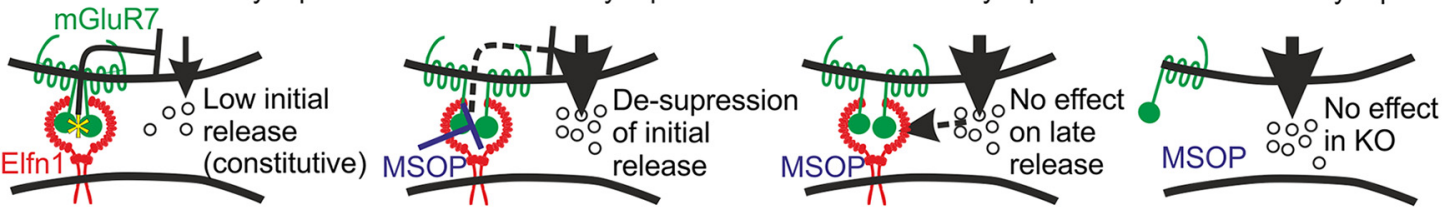
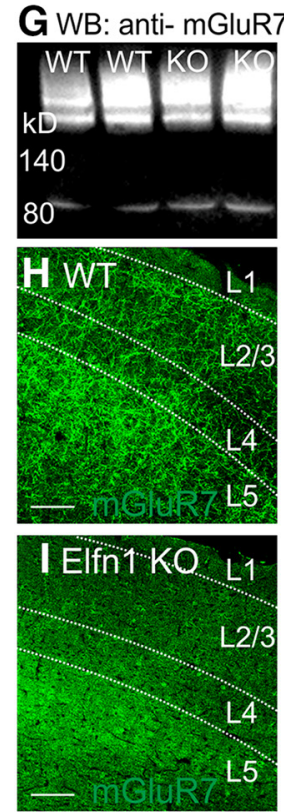

L Elfn1 WT SOM synapses

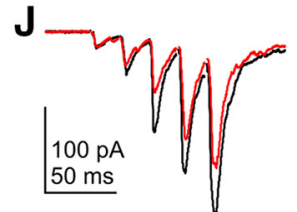

WT Baseline Low Dose L-AP4
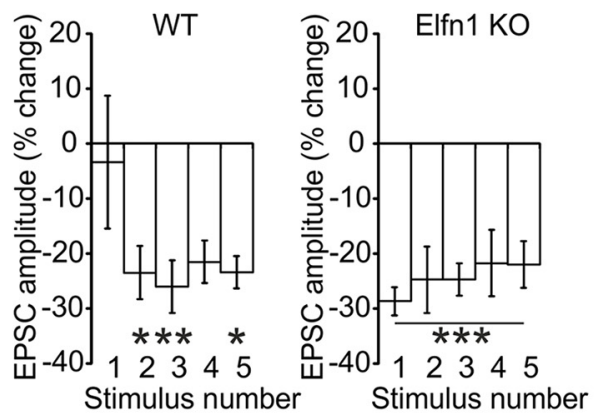
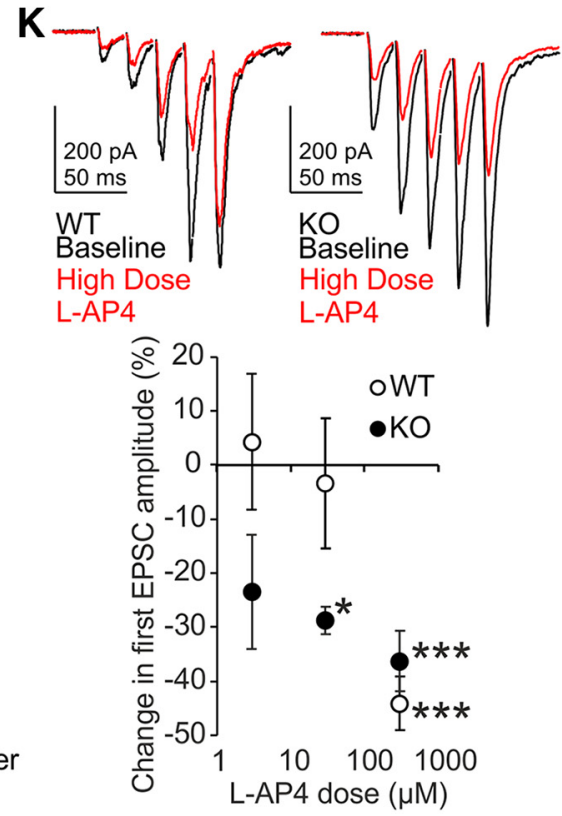

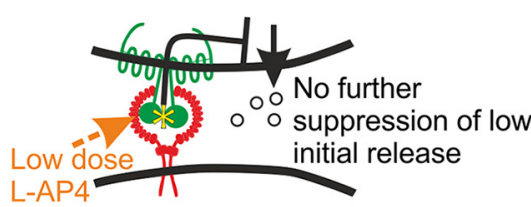

Elfn1 WT and KO SOM synapses

\section{Elfn1 WT and KO SOM synapses}

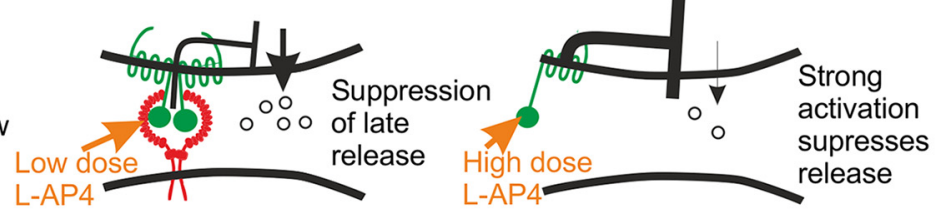

Figure 2. Elfn1 produces constitutive suppression of synaptic transmission at pyr $\rightarrow$ SOM synapses through agonist-independent $\mathrm{mGluR7}$ activation. A, $\mathrm{mGluR7}$ (green) colocalizes with Elfn1 (red) at synapses in cortical neuronal cultures. B, Selective synaptic distribution of mGluR7 immunofluorescence (green) onto the dendritic arbor of a L2/3 SOM interneuron (red) in cortical slices. C, Colocalization of mGluR7 with dendritic arbors is disrupted in Elfn1 KO L2/3 SOM neurons. D, mGluR7 inhibition with MSOP (100 $\mu \mathrm{M})$ increases evoked EPSC amplitude onto L2/3 SOM neurons for the first EPSC. Initial release probability is increased. E, MSOP has no effect at L2/3 synapses in Elfn $1 \mathrm{KO}$. $\boldsymbol{F}$, Summary of MSOP findings. Elfn1-induced recruitment of mGluR7 induces constitutive activity, which suppresses synaptic release. This constitutive activity is antagonized by MSOP, desuppressing initial release. Surprisingly, no effect of blocking synaptic glutamate is detected, as late events are not altered by MSOP blockade. Likewise, no effect is detected in the absence of Elfn1. G, Absence of mGluR7 activity in Elfn $1 \mathrm{KO}$ is not due to reduction in total protein, as shown by Western blot of cortical lysates. $\boldsymbol{H}, \boldsymbol{I}$, Redistribution of mGluR7 protein. In Elfn $1 \mathrm{KO}, \mathrm{mGluR7}$ no longer labels dendritic processes. J, Application of the mGluR agonist L-AP4 (30 $\mu \mathrm{M}$ ) fails to further suppress initial release in WT but not in Elfn $1 \mathrm{KO}$ animals. Late release events are still affected. $K, L-A P 4(300 \mu \mathrm{M})$ is still able to suppress initial release in both WT and K0 animals. Partial dose-response profiles for L-AP4 diverge in WT and K0 animals. L, Summary of L-AP4 findings. Elfn1-induced constitutive activity occludes the suppression of initial release by L-AP4 in WT but not K0 animals. Agonism with exogenous L-AP4 unveils a canonical autoreceptor function not observed for endogenous glutamate. Data are mean \pm SEM. Scale bars: $A-C, 10 \mu \mathrm{m} ; \boldsymbol{H}, \boldsymbol{I}, 100 \mu \mathrm{m}$. ${ }^{*} p<0.05,{ }^{* * *} p<0.001$. n.S., Not significant $(p>0.05)$. 
pA in $300 \mu \mathrm{M} \mathrm{L}-\mathrm{AP} 4 \mathrm{vs} 51 \pm 11 \mathrm{pA}$ at baseline, $p=0.0004, t=$ 4.90, paired $t$ test, $n / N=13 / 6, \mathrm{~L} 2 / 3 \mathrm{KO}: 37 \pm 8$ in $300 \mu \mathrm{M} \mathrm{L}-\mathrm{AP} 4$ vs $65 \pm 18$ at baseline, $p=0.02, t=2.82$, paired $t$ test, $n / N=9 / 4$; Fig. $2 \mathrm{~K})$. Comparing responses across doses and genotypes, we find that initial release in WT, L-AP4 $(300 \mu \mathrm{M}), \mathrm{KO}, \mathrm{L}-\mathrm{AP} 4(300$ $\mu \mathrm{M})$, and $\mathrm{KO}, \mathrm{L}-\mathrm{AP} 4(30 \mu \mathrm{M})$ differ from baseline $(p=0.000001$, $p=0.0009$, and $p=0.03$, respectively, $F=4.4$, two-way ANOVA, Bonferroni post hoc). In contrast to antagonism with MSOP, later EPSCs in both WT and KO slices showed synaptic suppression in the presence of exogenous agonist (change vs baseline, WT: EPSC $1, p=1$; EPSC2, $p=0.02$; EPSC $3, p=0.006$; EPSC $4, p=$ 0.06; EPSC5, $p=0.03 ; F=44.31$, two-way ANOVA, post hoc Bonferroni, $n / N=11 / 5$; change vs baseline, KO: EPSC1, $p=9 \times$ $10^{-7}$; EPSC2, $p=3 \times 10^{-5} ;$ EPSC $3, p=3 \times 10^{-5}$; EPSC4, $p=$ 0.0004; EPSC5, $p=0.0004, F=140.18$, two-way ANOVA, post hoc Bonferroni, $n / N=9 / 2$ ). Thus, although mGluR7 can act as an "autoreceptor" in response to exogenous application of L-AP4, we find no evidence for autoreceptor activity in the generation of synaptic facilitation at pyr $\rightarrow$ SOM synapses (Fig. $2 L$ ).

\section{Elfn1 recruitment of $\mathrm{mGluR7}$ generates constitutive mGluR7 activity}

We tested the sufficiency of Elfn1 to generate constitutive mGluR7 activity in a heterologous cell assay. HEK cell tight junctions consist of transcellular interactions between proteins, with the close proximity of the interacting membranes affording an opportunity for protein-protein interactions to preferentially localize interacting partners to junctions (Williams et al., 2011). By transfecting mGluR7-GFP into one population of cells and Elfn 1tdTomato into another population of cells, then coplating the cells, we were able to assess the transcellular interaction between Elfn 1 and mGluR7 at tight junctions by measuring GFP fluorescence intensity (Fig. 3A). Cells expressing mGluR7-GFP displayed an $\sim 6$-fold greater fluorescence intensity at tight junctions (defined by ZO1 label) of juxtaposed cells expressing Elfn1tdTomato than at tight junctions formed with Elfn1-tdTomatonegative cells, indicating that Elfn1 is sufficient to cluster mGluR7 via a transcellular interaction (mGluR7-GFP fluorescence intensity: $6.1 \pm 1.1$-fold increase at Elfn 1 junctions vs nonElfn1 junctions, $p=3 \times 10^{-9}, t=7.22$, paired $t$ test, $n / N=$ $50 / 8)$. Removal of the extracellular ligand binding domain of mGluR7 prevented the Elfn1-induced recruitment ( $\Delta$ ectomGluR7-GFP intensity: $1.0 \pm 0.2$-fold increase at Elfn 1 junctions, $p=0.1, t=1.74$, paired $t$ test, $n / N=21 / 3$ ), as did removal of the LRR binding domain for Elfn1 (mGluR7-GFP intensity: $1.1 \pm 0.1$-fold increase at Elfn1- $\Delta$ LRR junctions, $p=0.3, t=$ 1.18, paired $t$ test, $n / N=19 / 3$; mGluR7-GFP/Elfn 1 conditionvs $\Delta$ ecto-mGluR7-GFP/Elfn 1 condition, $p=0.003$, vs mGluR7GFP/ $\Delta$ LRR-Elfn 1 condition, $p=0.006, F=8.38$, one-way ANOVA, post hoc Bonferroni; Fig. $3 B$ ). The absence of mGluR7 enrichment in the deletion constructs was not due to mislocalization of the mutant proteins (data not shown).

We next assessed whether transcellular clustering of mGluR7 might have functional consequences for receptor activation. Dimerization of $\mathrm{G}_{\mathrm{i}} / \mathrm{G}_{\mathrm{o}}$-coupled metabotropic glutamate receptors induces a weak, constitutive activation of inwardly rectifying potassium channel (GIRK) conductance through a conformational change in the ligand binding domain that bears similarities to agonist binding (Levitz et al., 2016). Noting that Elfn 1 is present in a multimeric form (Fig. $3 C$ ), we hypothesized that the clustering induced by Elfn 1 could constitutively activate mGluR7 by stabilizing dimerized mGluR7 in an active conformation. As a sensitive assay for mGluR7 activation, we measured changes in potassium conductance in HEK cells, cotransfected with mGluR7-GFP and GIRK1/2, in response to application of two soluble putative dimerizing agents: MAB1/28 and Elfn-Fc. We used MAB1/28, an antibody directed against the extracellular domain of mGluR7 that immunoprecipitates (IP; Fig. 3D) and selectively binds to dimeric mGluR7 (Ullmer et al., 2012), to determine whether receptor dimer stabilization alone is sufficient to induce GIRK activation. We used Elfn-Fc, a soluble Elfn dimer composed of the extracellular domain of Elfn 1 fused to an Fc domain, to mimic Elfn1-induced dimer stabilization (Fig. 3E). Focal application of MAB1/28 to mGluR7-expressing HEK cells produced a reversible increase in GIRK (Fig. $3 F$ ). Conductance was calculated from the current response to a series of voltage steps between -125 and $15 \mathrm{mV}$, which enabled intrinsic verification that the dimerization-activated conductance was an inwardly rectifying potassium conductance (GIRK; Fig. 3G). In contrast, application of a control antibody had no effect (conductance: $1.34 \pm 0.38 \mathrm{nS}$ with control antibody vs $1.35 \pm 0.39 \mathrm{nS}$ baseline, $p=0.4, t=0.92$, paired $t$ test, $n / N=5 / 1$; conductance: $1.01 \pm 0.38 \mathrm{nS}$ with MAB1/28 vs $0.92 \pm 0.38 \mathrm{nS}$ baseline, $p=$ $0.02, t=3.27$, paired $t$ test, $n / N=7 / 4$; Fig. $3 H$ ). Focal application of Elfn-Fc likewise produced an increase in GIRK, suggesting that Elfn1-mediated dimer stabilization of mGluR7 is sufficient to increase receptor activity. In contrast, control Fc protein had no effect (conductance: $0.98 \pm 0.25 \mathrm{nS}$ with control Fc vs $0.98 \pm 0.25$ $\mathrm{nS}$ baseline, $p=0.5, t=0.70$, paired $t$ test, $n / N=10 / 4$; conductance: $1.26 \pm 0.45 \mathrm{nS}$ with Elfn $1-\mathrm{Fc}$ vs $1.11 \pm 0.39 \mathrm{nS}$ baseline, $p=0.04, t=2.42$, paired $t$ test, $n / N=11 / 6$; Fig. $3 I$ ). Notably, MAB1/28 reportedly does not induce detectable $G_{i} / G_{o}$ activation, as assessed by suppression of forskolin-induced cAMP activation, or by calcium mobilization with promiscuous G-protein coupling in a FLIPR assay (Ullmer et al., 2012). To test whether Elfn1 likewise had biased signaling properties regarding $G_{i} / G_{0}$ activation, we assessed $\mathrm{G} \alpha$-mediated calcium mobilization with a FLIPR assay validated with a canonical mGluR7 activator, L-AP4 (Fig. 3J) (Lindemann et al., 2011). As with MAB1/28, Elfn1-Fc protein did not induce detectable $\mathrm{G} \alpha$ signaling as measured by FLIPR (Fig. $3 K$ ), suggesting a divergence from the diffusible second messenger signaling cascades typically evoked by agonist activation. Indeed, a recent study demonstrates that Elfn 1 interactions produce negative allosteric modulation of Type III mGluRs by suppressing G-protein coupling efficiency for $\mathrm{G} \alpha$ and $\mathrm{G} \alpha_{\mathrm{o}}$ (Dunn et al., 2018). This series of observations is instead consistent with the fast, membrane delimited, voltage-sensitive suppression of calcium channels by G-protein coupled receptors (GPCRs) described by Hille and colleagues (Beech et al., 1992). Evidence suggests that mGluR7 homodimerization likewise induces a constitutive, voltage-sensitive suppression of calcium channel activity (Kammermeier, 2015).

\section{Constitutive mGluR7 activity permits allosteric modulation of spike timing}

A voltage-dependent suppression of calcium channel activity predicts that synaptic suppression is transient within a train of action potentials, which may modulate SOM interneuron spike timing and thus impact circuit function. The constitutive mGluR7 activity present at pyr $\rightarrow$ SOM synapses could therefore allow for the use of allosteric modulators to impact the spike output of SOM neurons. Positive allosteric modulators enhance ongoing receptor activity, without generating the exogenous receptor activation and subsequent synaptic suppression produced by canonical agonists, such as L-AP4. We applied the mGluR7selective mixed agonist/positive allosteric modulator, AMN082, 


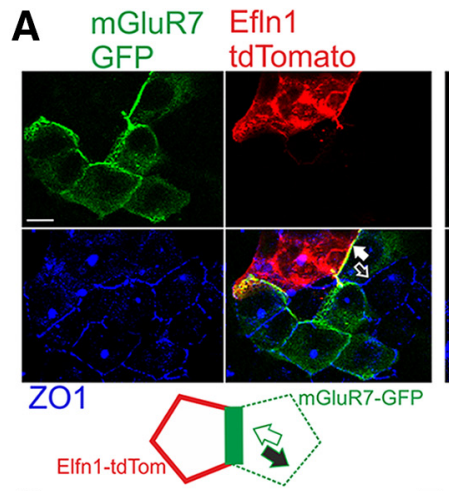

C

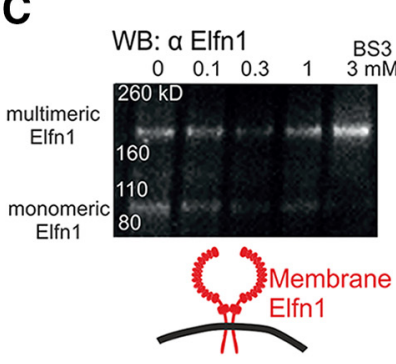

$\mathbf{F}$

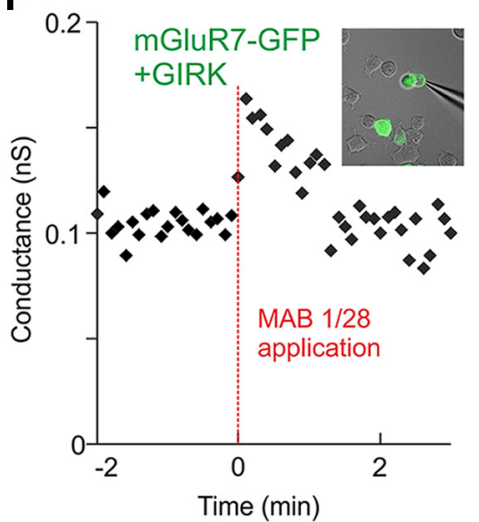

$\triangle$ ecto-mGluR7 Efln1

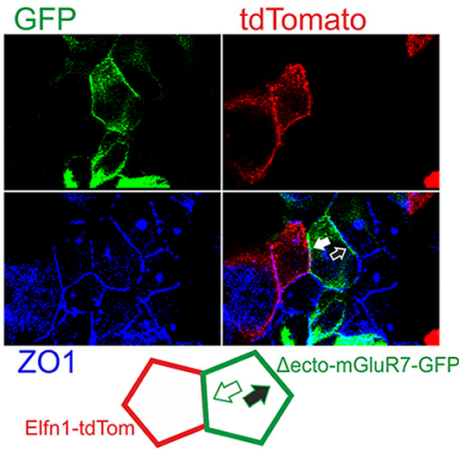

D

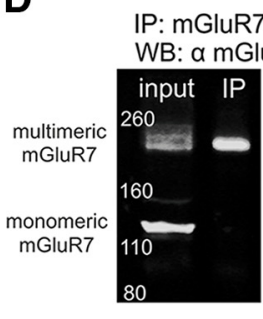

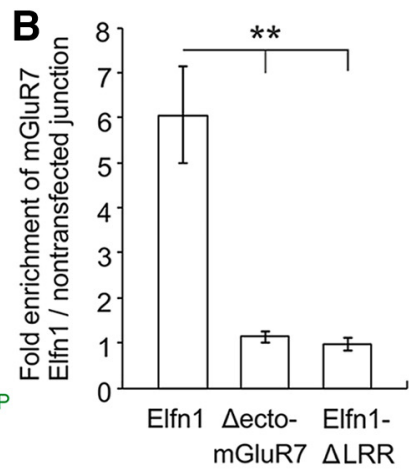

E

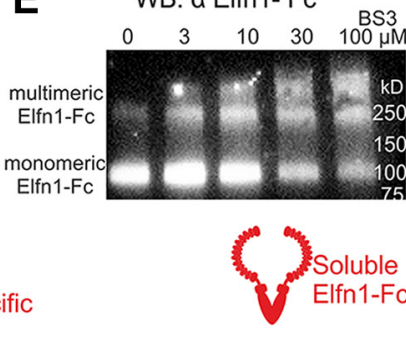

MAB1/28: dimer-specific antibody

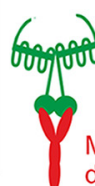

G

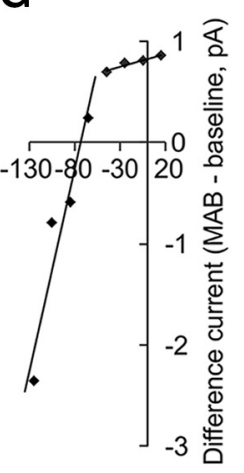

Holding Voltage $(\mathrm{mV})$
H
J

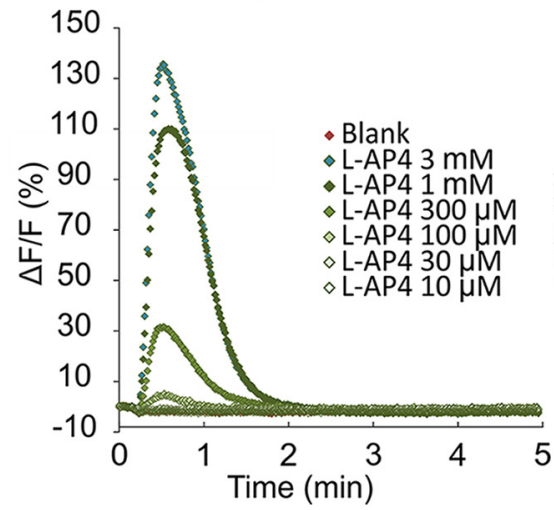

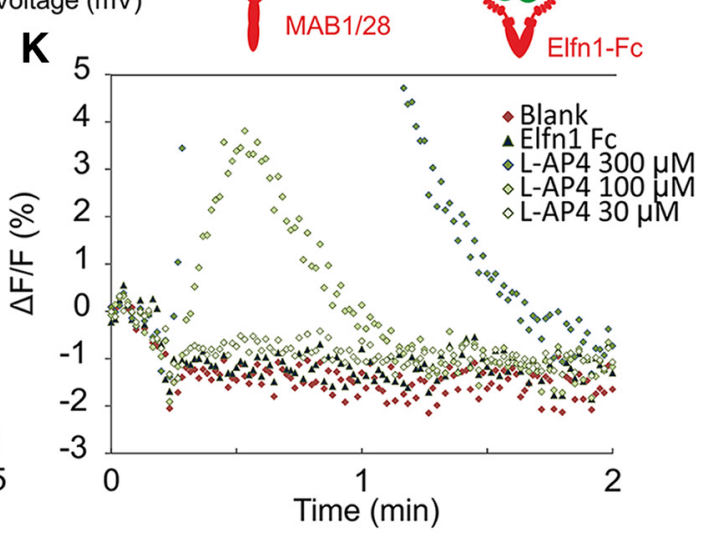

Figure 3. Elfn1-induced clustering of mGluR7 produces constitutive receptor activity. A, In HEK cell cultures, mGluR7-GFP intensity (green) is strongly enriched at Z0-1 (blue)-labeled cell-cell interfaces (white arrow) that are abutting Elfn1-containing cells (red), relative to interfaces abutting other cells (black arrow). In the absence of the extracellular domain of mGluR7 ( $\Delta$ ecto-mGluR7GFP), enrichment does not occur. B, Quantification of Elfn1 enrichment for mGluR7-GFP, $\Delta$ ecto-mGluR7-GFP, and for mGluR7-GFP abutting LRR-deleted versions of Elfn1. C, Elfn1 forms homomultimers, as evident from the increase in dimer band in lysates from Elfn1-transfected HEK cells treated with increasing concentrations of BS3 crosslinking agent. D, The mGluR7 antibody MAB1/28 specifically recognizes dimerized mGluR7, as immunoprecipitation (IP) with MAB1/28 preferentially pulls down dimerized mGluR7. Blotting with polyclonal anti-mGluR7 recognizes both monomer and dimer bands. $\boldsymbol{E}$, Elfn1-Fc exists as a dimer, based on Western blot analysis with BS3 crosslinking of purified protein samples. $\boldsymbol{F}$, Application of MAB1/28 produces an increase in conductance recorded from mGluR7/GIRK 1/2-transfected HEK cells. An example trace shows the time course in response to acute application. G, Current-voltage analysis of the induced conductance increase verifies that the activated channel is an inwardly rectifying (flattened $I-V$ above $-40 \mathrm{mV}$ ) potassium conductance (reversal near $-82 \mathrm{mV} ; \mathrm{K}^{+} \mathrm{E}_{\text {rev }}$ ). $\boldsymbol{H}, \mathbf{I}$, Quantitation shows the increase in $\mathrm{GIRK}$ induced by MAB1/28 $(\boldsymbol{H})$ or Elfn- $\mathrm{Fc}(\boldsymbol{I})$ as a percentage of the whole-cell conductance. The respective control treatments produced no increase. $J$, L-AP4 control produces robust agonism of $m G$ luR7, generating $\mathrm{G} \alpha$-mediated calcium fluorescence $(\Delta \mathrm{F} / \mathrm{F})$ in a FLIPR assay. An example trace is shown. Each point represents an individual $\Delta \mathrm{F} / \mathrm{F}$ measurement. $\boldsymbol{K}$, Elfn1-Fc shows no detectable $\mathrm{G} \alpha$-mediated fluorescence signal in a FLIPR assay. Data are mean \pm SEM. Scale bars, $10 \mu \mathrm{m} .{ }^{*} p<0.05,{ }^{* *} p<0.01$. 
A

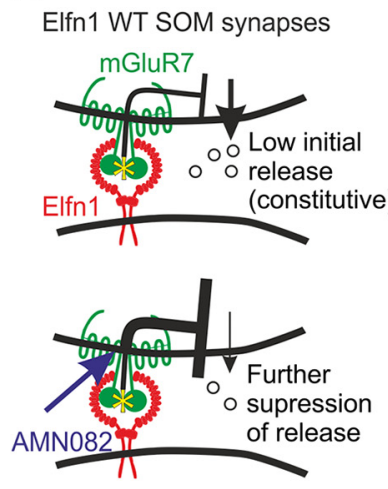

D Stimulus number

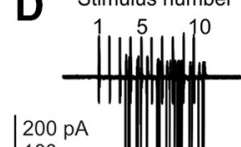

$200 \mathrm{pA}$

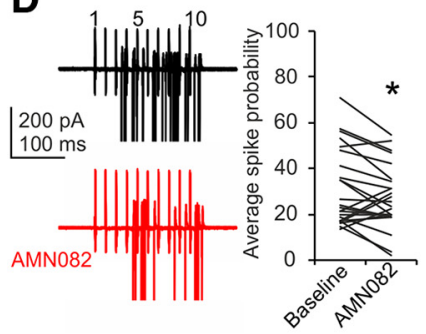

B mGluR7 allosteric modulation in WT L2/3 SOM neurons

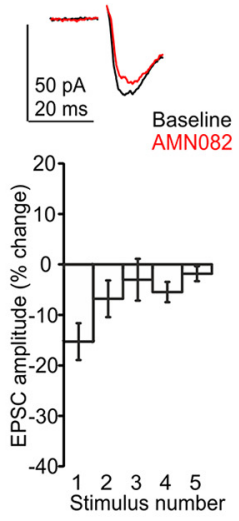

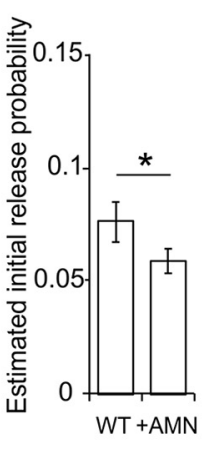
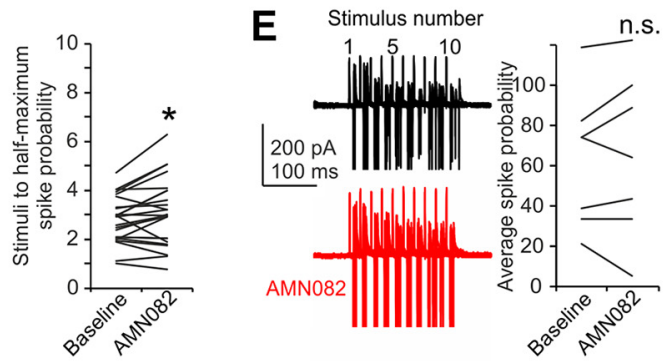

C mGluR7 allosteric modulation
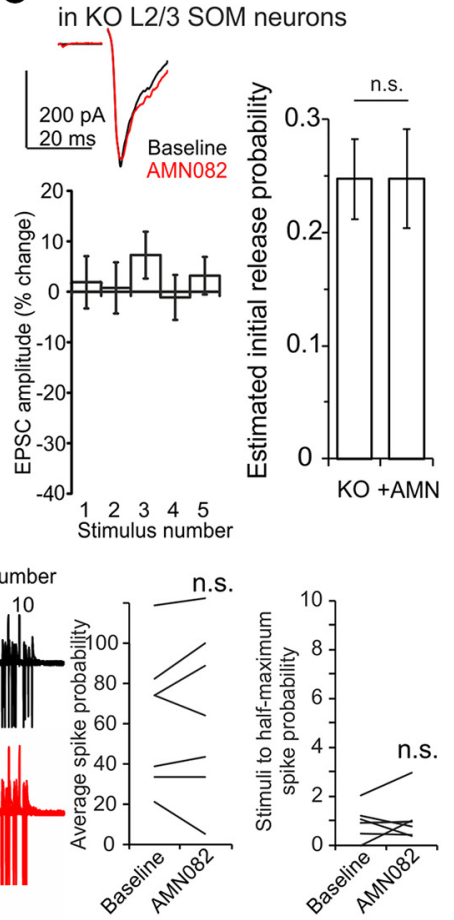

Figure 4. Constitutive mGluR7 activity permits allosteric modulation of spike timing at pyr $\rightarrow$ SOM synapses. $A$, Schematic illustration of AMN082 modulation of Elfn1-mGluR7 signaling. Elfn1 stabilization of an mGluR7 dimer active state induces constitutive activity, which suppresses synaptic release. AMN082 facilitates the constitutive activity of mGluR7, further suppressing release. $\boldsymbol{B}$, Activation of mGluR7 with $30 \mathrm{~nm}$ of the mixed agonist/positive allosteric modulator (PAM) AMN082 reduces early EPSC amplitude for L2/3 SOM neurons. AMN082 reduces initial release probability. C, AMN082 has no effect in Elfn 1 KO. D, AMN082 reduces spike probability and increases the number of stimuli needed to evoke to half-maximum spiking for synaptic-evoked spikes in L2/3 SOM neurons. Ten overlaid spike trains before (black) and during AMN082 wash in (red). E, Synaptic-evoked spiking shows little delay in Elfn $1 \mathrm{KO}$, and PAM activity of AMN082 is absent. Data are mean \pm SEM. ${ }^{*} p<0.05$. n.S., Not significant $(p>0.05)$.

at a low dose (30 nM; Fig. 4A), as at higher doses, AMN082 has been found to have a number of off-target effects (Mitsukawa et al., 2005; Sukoff Rizzo et al., 2011). We confirmed that low-dose AMN082 had effects on first EPSC amplitude, which we attribute to allosteric modulation of constitutively active mGluR7 by contrasting the effects of AMN082 with that of low-dose L-AP4 agonism (i.e., early suppression vs late suppression). The effect on initial release was absent in Elfn1 KO. AMN082 selectively impaired first EPSC amplitude at L2/3 synapses (EPSC amplitude: $33 \pm 6 \mathrm{pA}$ in AMN082 vs $37 \pm 6 \mathrm{pA}$ baseline, $p=0.002, t=4.65$, paired $t$ test, $n / N=8 / 4$; Fig. $4 B$ ). At L2/3 synapses in Elfn $1 \mathrm{KO}$ slices, $30 \mathrm{~nm}$ AMN082 failed to alter first EPSC amplitude (EPSC amplitude: $142 \pm 32 \mathrm{pA}$ in AMN082 vs $134 \pm 25 \mathrm{pA}$ baseline, $p=$ $0.5, t=0.79$, paired $t$ test, $n / N=9 / 2$; Fig. $4 C$ ).

We next assessed the effect of mGluR7 on synaptically induced SOM firing. Application of $30 \mathrm{~nm}$ AMN082 reduced average spike probability, and increased the number of stimuli required to reach half-maximal spiking in L2/3 neurons (spike probability, L2/3: $27 \pm 3 \%$ in AMN082 vs $31 \pm 3 \%$ at baseline, $p=0.04, t=2.14$, paired $t$ test; stimuli to half-maximum spiking, L2/3: $3.1 \pm 0.3$ in AMN082 vs $2.8 \pm 0.2$ at baseline, $p=0.03, t=$ 2.29, paired $t$ test, $n / N=24 / 4$; Fig. $4 D$ ). L2/3 KO neurons did not show impairments (spike probability, L2/3 KO: $65 \pm 15 \%$ in AMN082 vs $63 \pm 13 \%$ at baseline, $p=0.7, t=0.45$, paired $t$ test; stimuli to half-maximum spiking, L2/3 KO: $1.1 \pm 0.3$ in AMN082 vs $1.0 \pm 0.2$ at baseline, $p=0.6, t=0.52$, paired $t$ test, $n / N=7 / 2$; Fig. $4 E$ ). Thus, Elfn 1 induction of constitutive mGluR7 activity suppresses initial release probability, resulting in delayed recruitment of SOM interneurons in response to circuit activity. Positive allosteric modulation of mGluR7 can further delay SOM interneuron recruitment.
Constitutive mGluR7 activity generates frequency-dependent SOM synapses

Delayed recruitment of SOM interneurons in response to incoming synaptic input provides a possible explanation for the observation that the recruitment of hippocampal SOM neurons is frequency-dependent (Sun and Dobrunz, 2006). Elfn1 immunolabeling is visible in hippocampus and throughout the cortex (Fig. 5A). Initial release probability at $\mathrm{pyr} \rightarrow$ SOM synapses was similar across brain regions and was similarly increased in Elfn 1 $\mathrm{KO}$ animals across regions (L2/3 release probability, as in Fig. 1E: $0.19 \pm 0.02$ in $\mathrm{KO}$ vs $0.08 \pm 0.01$ in WT, $p=6 \times 10^{-5}, n / N=$ $22 / 5$ and 23/9, respectively; L5 release probability: $0.17 \pm 0.02$ in $\mathrm{KO}$ vs $0.10 \pm 0.01$ in WT, $p=0.03, n / N=20 / 11$ and $23 / 13$, respectively; $\mathrm{HC}$ release probability: $0.22 \pm 0.07$ in $\mathrm{KO}$ vs $0.07 \pm$ 0.01 in WT, $p=0.007, n / N=6 / 4$ and $11 / 5$, respectively; $F=$ 41.84, two-way ANOVA, post hoc Bonferroni; Fig. $5 B$ ). To assess frequency dependence of SOM synapses, we recorded synaptic events from SOM neurons in L2/3 in response to 5, 10, 20, 40, and $50 \mathrm{~Hz}$ synaptic stimulation $(50 \mathrm{~Hz}$ facilitation as in Fig. $1 B$, shown again for comparison in Fig. 5C). Short-term facilitation was impacted across a range of stimulus frequencies, with the most prominent disparity between WT and $\mathrm{KO}$ occurring at higher frequencies (L2/3 WT: $20 \mathrm{~Hz}, p=0.001,40 \mathrm{~Hz}, p=2 \times 10^{-10}, 50$ $\mathrm{Hz}, p=3 \times 10^{-17}$ vs KO, $F=69.77$, two-way ANOVA, post hoc Bonferroni, $n / N=21 / 4 \mathrm{WT}$ and $8 / 2 \mathrm{KO}$; Fig. $5 D$ ). L5 pyr $\rightarrow$ SOM synapses facilitation was also impacted by Elfn1 KO (EPSC5/ EPSC1 ratio: $3.4 \pm 0.3$ vs $4.6 \pm 0.4$ in WT, $p=0.02, t=2.46, t$ test, $n / N=31 / 13$ and 25/10, respectively; Fig. $5 E$ ). Recordings from L2/3 and L5 of cortex, as well as the CA1 region of the hippocampus, verified that release probability and frequency dependence generalize across SOM neurons (L5 EPSC5/EPSC1 ra- 

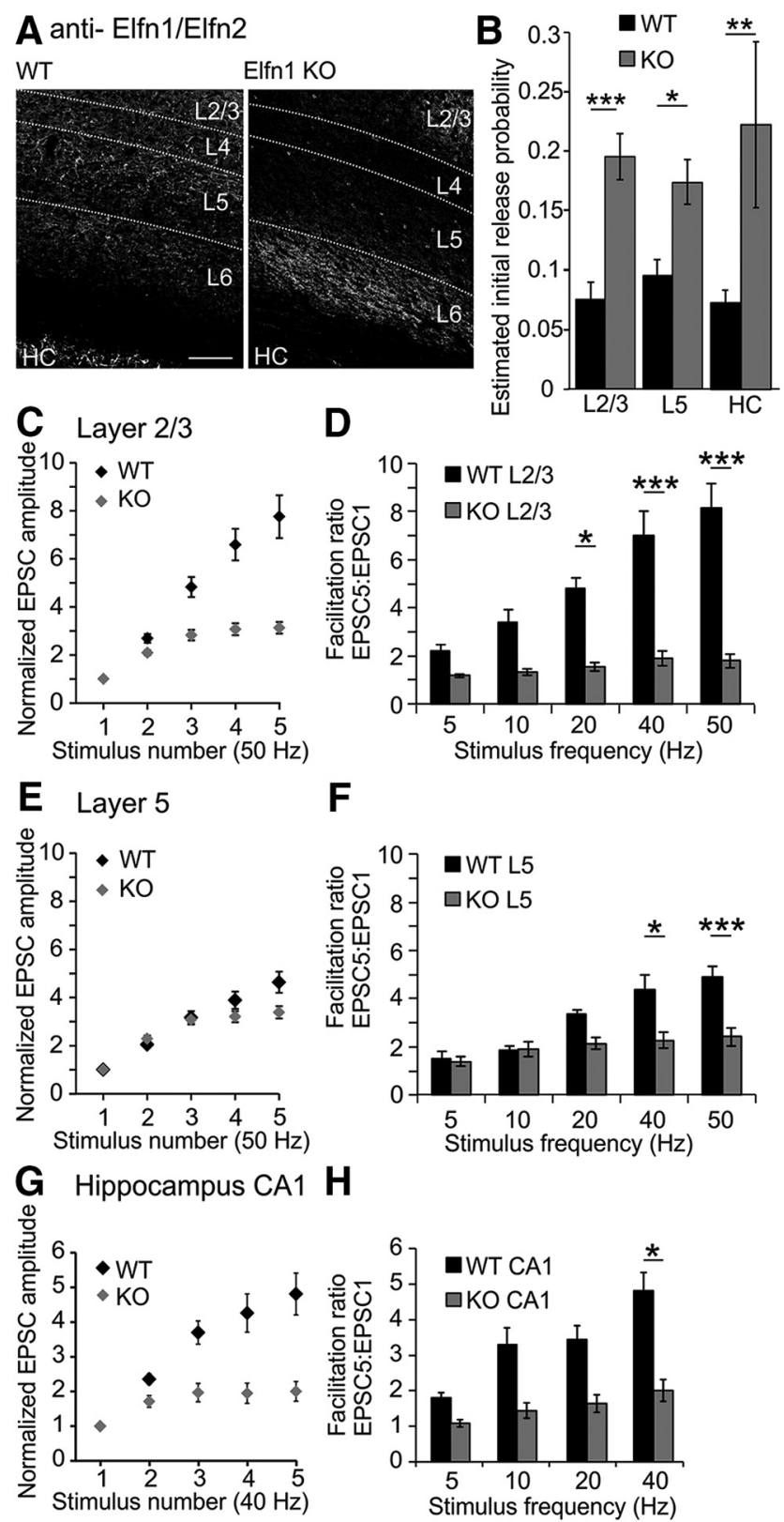

H

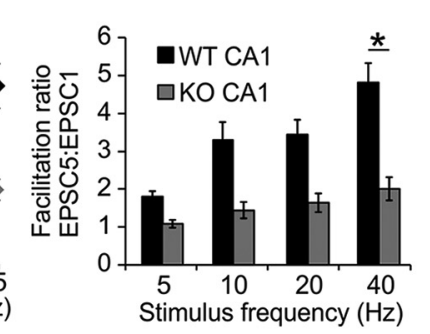

Figure 5. Elfn1 mediates release probability and frequency dependence of facilitation at SOM synapses. $\boldsymbol{A}$, Elfn1 immunostaining is visible in hippocampus and across cortical layers. Although anti-Elfn1/Elfn2 antibody is not specific for Elfn1, loss of staining on discrete dendritic processes in Elfn $1 \mathrm{KO}$ suggests that most of this signal is Elfn1. B, Release probability in WT (black) and KO (gray) SOM neurons is comparable across L2/3, L5, and hippocampus. C, In L2/3 of cortex, Elfn $1 \mathrm{KO}$ reduces facilitation ratio at $50 \mathrm{~Hz}$. Replicated from Figure 1, for comparison. $D$, Frequency-dependent facilitation in L2/3 increases at higher frequencies for WT (black) but not Elfn $1 \mathrm{KO}$ (gray) neurons. $\boldsymbol{E}$, Elfn $1 \mathrm{KO}$ has a less dramatic effect on $\mathrm{L} 5$ synaptic facilitation. $\boldsymbol{F}$, As in $L 2 / 3$, frequency-dependent facilitation in $L 5$ increases for WT (black) but not Elfn1 $\mathrm{KO}$ (gray) neurons. G, The presence of Elfn1 in SOM interneurons also produces a strongly facilitating synapse in hippocampus. Facilitation of EPSCs evoked in CA1 SOM neuron by alveus stimulation is attenuated in Elfn1 KO. $\boldsymbol{H}$, Elfn1 $\mathrm{KO}$ neurons (gray) show a frequency-dependent impairment in short-term facilitation, with the difference from WT (black), more pronounced for higher-frequency stimulation. Data are mean \pm SEM. ${ }^{*} p<0.05,{ }^{* *} p<0.01,{ }^{* * *} p<$ 0.001 .

tio: $40 \mathrm{~Hz}, p=0.002,50 \mathrm{~Hz}, p=2 \times 10^{-6}$ vs $\mathrm{KO}, F=17.68$, two-way ANOVA, post hoc Bonferroni, $n / N=15-17 / 7$ WT and 11/3 KO; Fig. 5F; CA1 EPSC5/EPSC1 ratio: $2.0 \pm 0.3$ in $\mathrm{KO}$ vs $4.8 \pm 0.6$ in WT, $p=0.0007, t=4.21, t$ test, $n / N=6 / 4$ and $13 / 5$, respectively; Fig. 5G; CA1: $40 \mathrm{~Hz}$ stimulation, $p=0.02, F=$ 24.30, two-way ANOVA, post hoc Bonferroni, $50 \mathrm{~Hz}$ was not acquired, $n / N=9-15 / 5 \mathrm{WT}$ and $6-7 / 4 \mathrm{KO}$; Fig. $5 H$ ). Notably, L5 pyr $\rightarrow$ SOM synaptic facilitation was less impacted by Elfn 1 $\mathrm{KO}$ than L2/3 (EPSC5/EPSC1 ratio at $50 \mathrm{~Hz}$ : layer $\times$ genotype interaction, $p=0.002, F=10.62$, two-way ANOVA).

\section{Constitutive mGluR7 activity at $\mathrm{pyr} \rightarrow$ SOM synapses is} similar in L2/3 and L5

SOM neurons in both L2/3 and L5 showed a strong short-term facilitation of excitatory transmission in response to highfrequency stimulation ( $50 \mathrm{~Hz}$; Fig. $6 A$ ), as seen previously for hippocampal SOM interneurons. However, L5 pyr $\rightarrow$ SOM synapses facilitated to a lesser extent (EPSC5/EPSC1 ratio: $4.6 \pm 0.4$

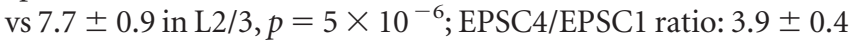
vs $6.6 \pm 0.7$ in $\mathrm{L} 2 / 3, p=0.0002, F=40.15$, two-way ANOVA, post hoc Bonferroni; $n=31 / 13$ and 24/10, respectively; L2/3 data as in Fig. $1 B, L 5$ data as in Fig. $5 E$, replotted for comparison in Fig. $6 B)$. L5 pyr $\rightarrow$ SOM synapses also showed longer delays in the synaptic induction of SOM interneuron spiking (stimuli to halfmaximum spiking: $4.7 \pm 0.3$ in L 5 vs $2.8 \pm 0.2$ in L2/3, corrected $p=4 \times 10^{-6}, t=5.31, t$ test, $n / N=24 / 4$ and $24 / 5$, respectively; $\mathrm{L} 2 / 3$ data as in Fig. $1 C, D$; replotted for comparison in Fig. $6 C, D$ ).

Similar to L2/3, L5 shows a punctate immunostaining pattern for mGluR7, concentrated at the dendrites of SOM neurons labeled with tdTomato (Fig. 6E). Further, MSOP increased synaptic transmission at pyr $\rightarrow$ SOM synapses in L5 (EPSC amplitude: $27 \pm 5 \mathrm{pA}$ in MSOP vs $21 \pm 3 \mathrm{pA}$ baseline, $p=0.04, t=-2.46$, paired $t$ test, $n / N=9 / 5$; Fig. $6 F$ ). Positive allosteric modulation of mGluR7 at L5 synapses reduced first EPSC amplitude (EPSC amplitude: $68 \pm 6 \mathrm{pA}$ in AMN082 vs $74 \pm 7 \mathrm{pA}$ baseline, $p=$ $0.009, t=3.31$, paired $t$ test, $n / N=10 / 3$; Fig. $6 G$ ). No difference in mGluR7 activity was detected, as measured by the change in synaptic release probability for L2/3 and L5 in response to MSOP or in response to AMN082 (Fig. $6 H$ ), although baseline facilitation ratios were different between L2/3 and L5 for those conditions $\left(p=1 \times 10^{-5}\right.$ for L2/3 vs L5 at baseline, $F=25.68$, two-way ANOVA). As an internal control for effects of synaptic rundown over time, comparison of MSOP effects against AMN082 effects on release probability significantly diverged from one another over the same time window (L2/3: $p=0.003$; L5: $p=0.02$ for MSOP vs AMN082, $F=24.69$, by two-way ANOVA, Bonferroni post hoc). As with L2/3 neurons, L5 neurons also showed reduced and delayed synaptic spiking in response to AMN082 (spike probability, L5: $23 \pm 4 \%$ in AMN082 vs $26 \pm 3 \%$ at baseline, $p=0.0005, t=4.07$, paired $t$ test; stimuli to halfmaximum spiking, L5: $5.4 \pm 0.5$ in AMN082 vs $4.7 \pm 0.3$ at baseline, $p=0.02, t=2.43$, paired $t$ test, $n / N=24 / 5$; Fig. $6 I$ ). Because mGluR7 activity and protein were detected at both L2/3 and L5 synapses, mGluR7 activity cannot explain the layerspecific differences in facilitation ratio.

\section{Elfn1 recruitment of GluK2-KARs is nonconstitutive but} generates a strongly facilitating synapse

The strong facilitation at hippocampal pyr $\rightarrow$ SOM synapses during natural stimulus patterns is attributed, in part, to the presence of calcium-permeable presynaptic kainate receptors (responsive to the GluK2-KAR selective antagonist, NS102; Fig. 7A) (Sun et al., 2009). Elfn 1 is necessary for recruitment of presynaptic GluK2-KARs, as shown by the loss of NS102 sensitivity following shRNA knockdown of Elfn1 (Sylwestrak and Ghosh, 2012). The presence of constitutive mGluR7 activity at both L2/3 and L5 synapses left us with the hypothesis that the layer-specific differ- 
A Cortex

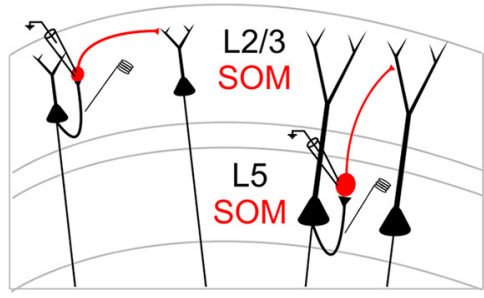

D

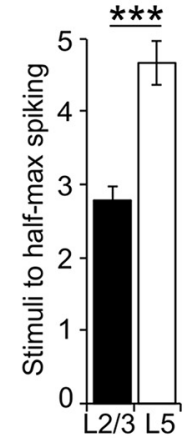

E
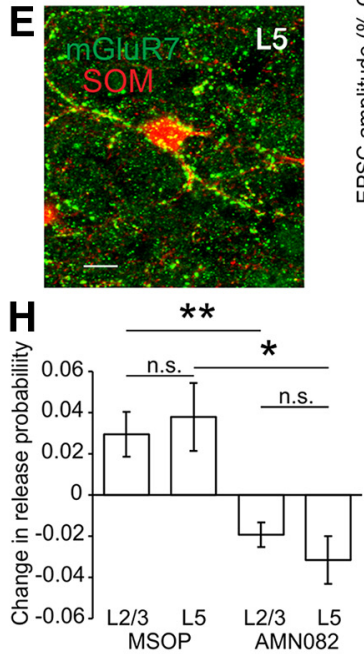

B

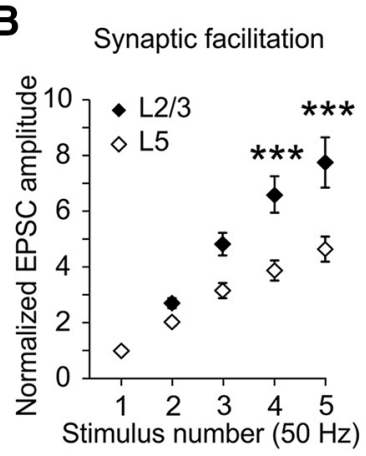

C 80 Synaptic-induced

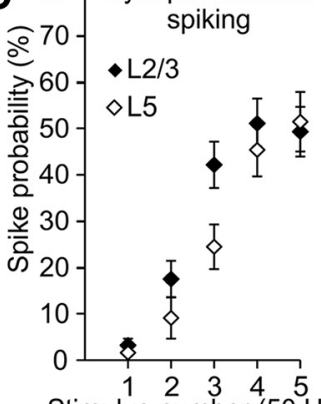

Stimulus number $(50 \mathrm{~Hz})$
F mGluR7 blockade in WT L5 SOM neurons

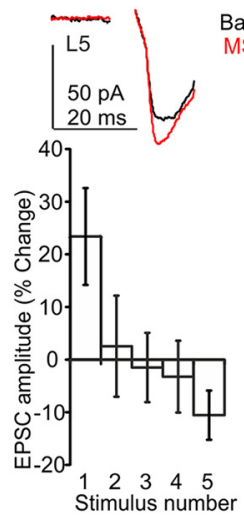

Baseline
MSOP

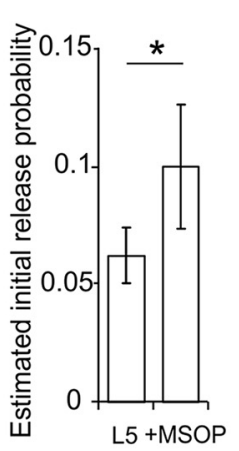

G mGluR7 allosteric modulation in WT L5 SOM neurons

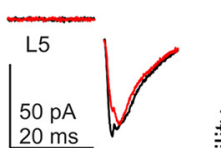

Baseline AMN082
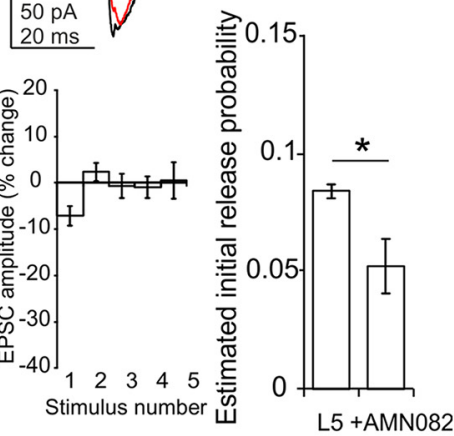

1
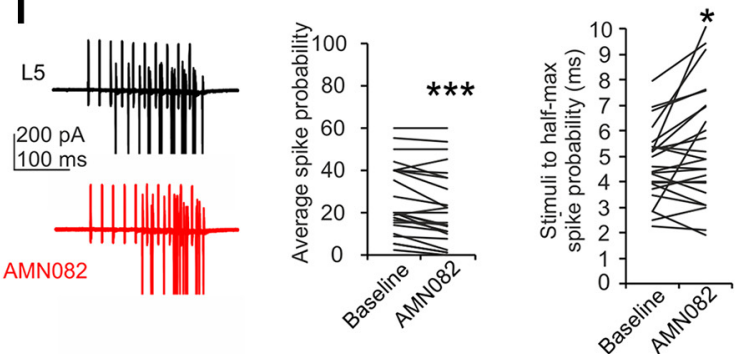

Figure 6. Constitutive $m G l u R 7$ activity at pyr $\rightarrow$ SOM synapses is similar in L2/3 and L5. A, Recording setup for L2/3 SOM neurons or L5 SOM neurons; stimulation is within layers. $\boldsymbol{B}$, In L2/3 and $\mathrm{L} 5$ of cortex, facilitation ratios at $50 \mathrm{~Hz}$ differ in magnitude. ESPC amplitude is normalized to first EPSC amplitude. C, Within a $50 \mathrm{~Hz}$ train of synaptic-driven SOM activation, spiking onset is delayed in both L2/3 and L5 neurons. D, Probability of spiking takes more stimuli to reach half-maximum in L5 compared with L2/3 neurons. $E$, $\mathrm{mGluR7}$ immunofluorescence (green) colocalizes with dendritic arbors of L5 SOM interneurons (red) in cortical slices. F, mGluR7 inhibition with MSOP (100 $\mu \mathrm{M})$ increases evoked EPSC amplitude onto L5 SOM neurons for the first EPSC. Initial release probability is increased. G, Activation of mGluR7 with $30 \mathrm{~nm}$ of the mixed agonist/positive allosteric modulator (PAM) AMN082 reduces early EPSC amplitude for L5 SOM neurons. Initial release probability is reduced. $\boldsymbol{H}$, The change in release probability in response to MSOP or AMN082 does not differ between L2/3 and L5. I, AMN082 reduces spike probability and increases the number of stimuli to half-maximum spiking for synaptic-evoked spikes in L5SOM neurons. Ten overlaid spike trains before (black) and during AMN082 wash in (red). Data are mean \pm SEM. Scale bar, $10 \mu \mathrm{m}$. ${ }^{*} p<0.05$, ${ }^{* *} p<0.01,{ }^{* * *} p<0.001$. n.s., Not significant $(p>0.05)$.

ences in facilitation ratio at these 2 synapses was the result of differences in presynaptic GluK2-KARs. We first verified that GluK2-KARs are present at cortical synapses by looking for cell type-specific localization on dissociated cortical neuronal cultures. Using an antibody that recognizes GluK2, but also GluK3, we found a strong colocalization with synaptic mGluR7 (Fig. 7B). However, the GluK2/3 staining was not as discretely localized as mGluR7, labeling other neurons and cell bodies as well. The presence of presynaptic GluK2-KARs generates a late, strong facilitation of glutamatergic synaptic transmission onto SOM cells in L2/3 cortex that is reduced by the GluK2-KAR antagonist NS102 $(20 \mu \mathrm{M}$, EPSC5/EPSC1, L2/3: $5.4 \pm 0.7$ in NS102 vs $6.7 \pm 0.9$ at baseline, $p=0.007, t=4.03$, paired $t$ test, $n / N=7 / 5$; Fig. 7C). The reversible change in synaptic transmission was primarily re- stricted to EPSCs evoked later in the train (EPSC5 amplitude: $318 \pm 68 \mathrm{pA}$ in NS102 vs $376 \pm 72 \mathrm{pA}$ at baseline, $p=0.001, t=$ 5.89 , paired $t$ test, $n / N=7 / 5$ ). In contrast to $\mathrm{L} 2 / 3$, we found that presynaptic GluK2-KARs do not play a role in the facilitation of excitatory synaptic inputs onto L5 SOM neurons, as these synapses facilitate to a lesser extent and are NS-102 insensitive (EPSC5/EPSC1, L5: $3.4 \pm 0.7$ in NS102 vs $3.4 \pm 1.3$ at baseline, $p=0.9, t=0.11$, paired $t$ test, $n / N=7 / 5$; Fig. $7 D$ ). L2 $/ 3 \mathrm{SOM}$ neurons from Elfn1 KO animals are also NS-102 insensitive (EPSC5/EPSC1, L2/3 KO: $0.8 \pm 0.1$ in NS102 vs $0.8 \pm 0.3$ at baseline, $p=0.5, t=0.65$, paired $t$ test, $n / N=6 / 1$; Fig. $7 E$ ). Coefficient of variation analysis (Faber and Korn, 1991) indicates that the NS102-sensitive receptors alter presynaptic release probability (EPSC5; Fig. 7F), consistent with previous studies 

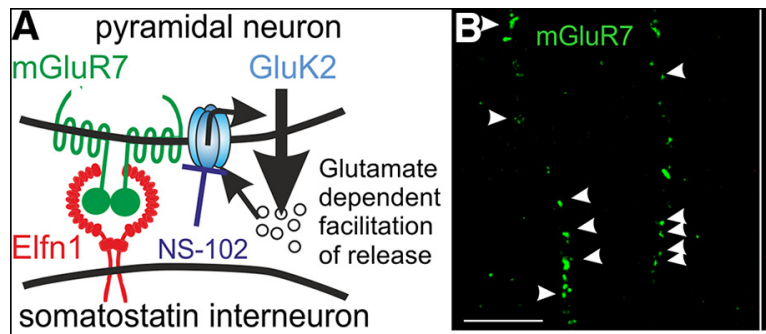

D Layer 5

\section{Layer $2 / 3$}

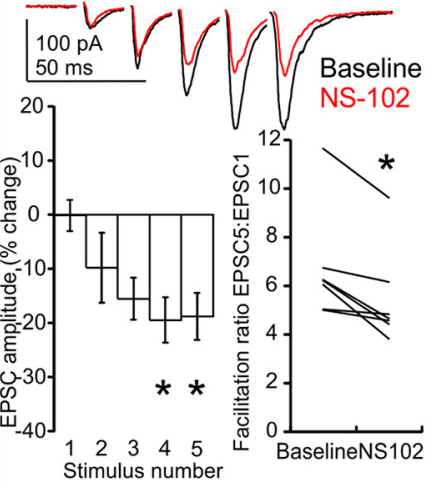

$\mathbf{F}$
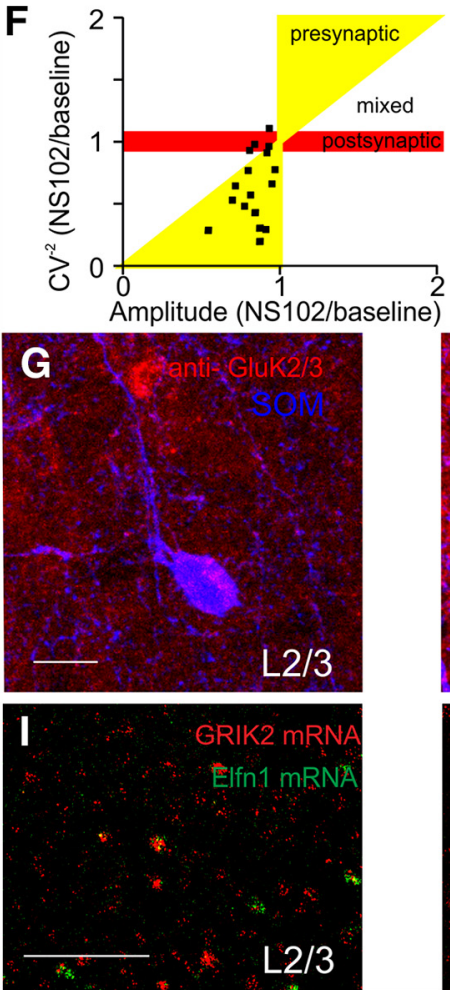

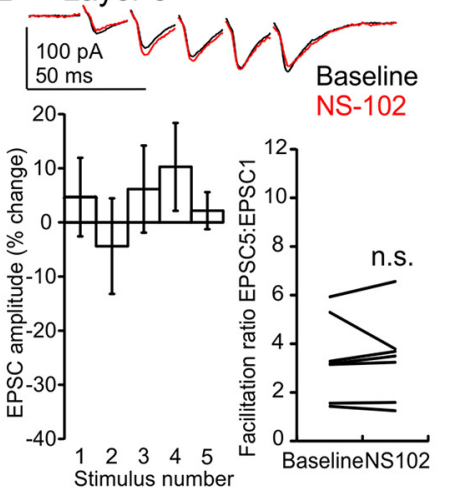

K cortical lysates WB: anti- GluK2/3
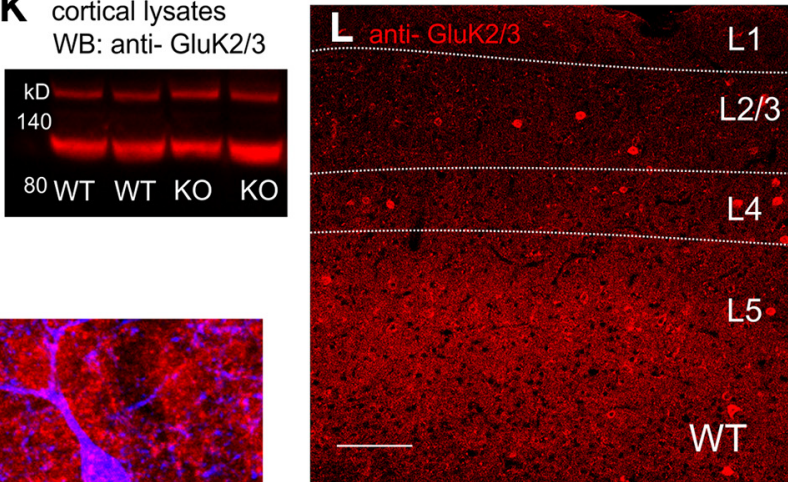

E Layer $2 / 3 \mathrm{KO}$
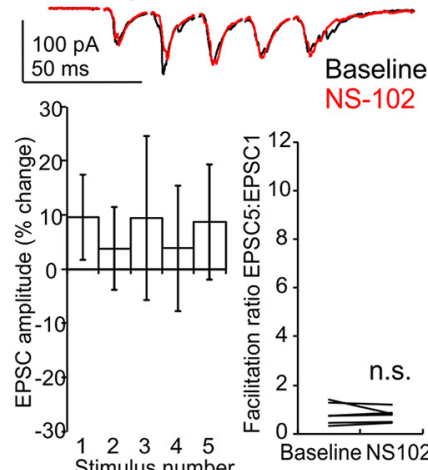

NS-102

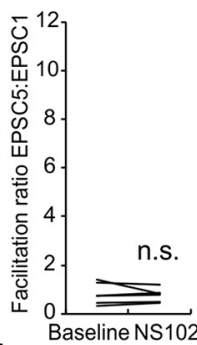

n.s.

Baseline NS10

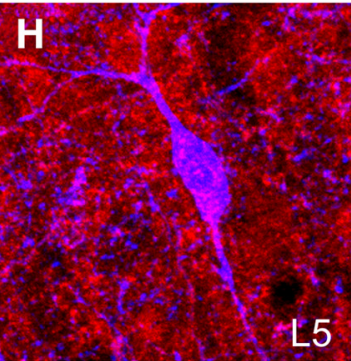

M

Figure 7. Strongly facilitating synaptic transmission at pyr $\rightarrow$ SOM synapses is mediated by recruitment of GluK2-containing kainate receptors (GluK2-KARs). $A$, Proposed organization of excitatory pyr $\rightarrow$ SOM synapses in L2/3, with Elfn1-clustered mGluR7 and presynaptic GluK2-KARs. B, GluK2/3 (aka GluR6/7) staining colocalizes with mGluR7 on dendrites of cortical neurons in culture (arrows). C, Facilitation onto L2/3 neurons is suppressed by NS-102, implicating GluK2-KARs. D, L5 to L2/3 differences can be explained by the absence of recruitment of GluK2-KARs in L5. $E$, NS-102 has no effect in Elfn 1 KO L2/3 neurons. Facilitation ratio in L2/3 WT, but not in L5WT nor in L2/3 K0, is altered by NS-102. F, Coefficient of variation analysis shows that the GluK2-selective antagonist NS-102 primarily alters synaptic amplitude (EPSC5) by changing presynaptic release probability (region in yellow), rather than postsynaptic amplitude (postsynaptic changes would lie along the horizontal; red). $\mathbf{G}, \boldsymbol{H}$, $\mathrm{GluK} 2 / 3$ immunostaining (red) is not reduced for L5 compared with L2/3 SOM neurons (blue). Staining is visible in presynaptic and postsynaptic cells. I,J, GRIK2 mRNA (red) labels an abundance of cells in both L2/3 and L5. Elfn $1 \mathrm{mRNA}$ labels a smaller subset of cells in these layers. $K$, GluK2/3 immunoblotting does not show reductions in total protein in Elfn $1 \mathrm{KO}$ cortical lysates compared with WT. $L, M$, GluK2/3 immunostaining does not show reductions in GluK2/3 in Elfn1 K0. Data are mean \pm SEM. Scale bars: $\boldsymbol{B}, \mathbf{G}, \boldsymbol{H}, 10 \mu \mathrm{m} ; \boldsymbol{I}, \mathbf{J}, \mathbf{L}, \boldsymbol{M}, 100 \mu \mathrm{m} .{ }^{*} p<0.05$. n.s., Not significant $(p>0.05)$.

indicating that, for excitatory synapses onto SOM neurons, GluK2-KARs are presynaptic and calcium-permeable (Sun and Dobrunz, 2006; Sun et al., 2009). The presence of GluK2 protein and mRNA in both L2/3 and L5 suggests that differences in
GluK2-KARs are not due to an absence of GluK2 in L5 (Fig. $7 G-J$ ). Likewise, no reduction in GluK2 expression levels were observed in $\mathrm{KO}$ animals compared with WT by Western blot or immunostaining (Fig. $7 \mathrm{~K}-\mathrm{M}$ ), although the strong postsynaptic 


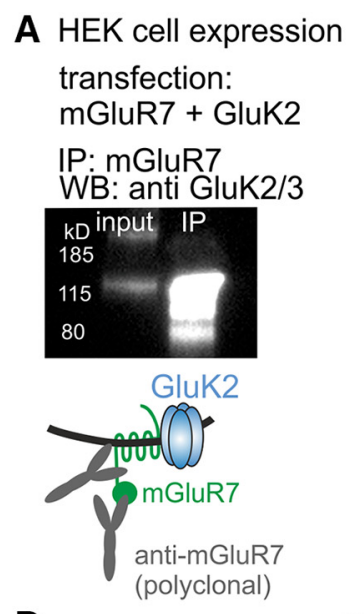

B cortical lysates
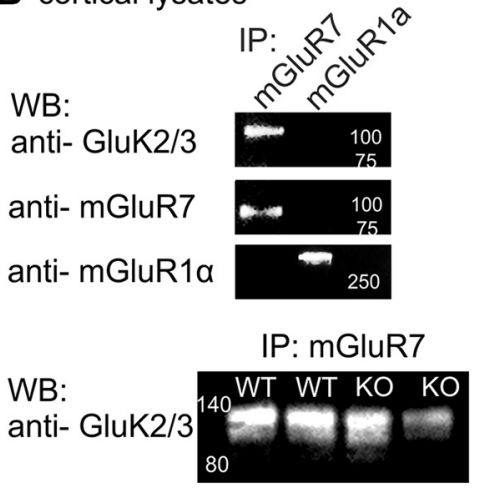

\section{Layer 5, Calmodulin activated}

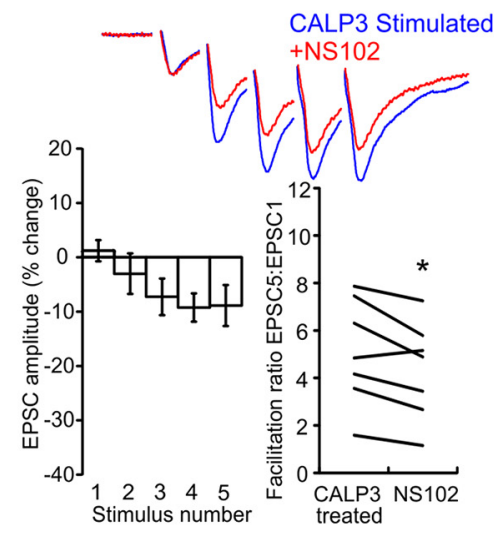

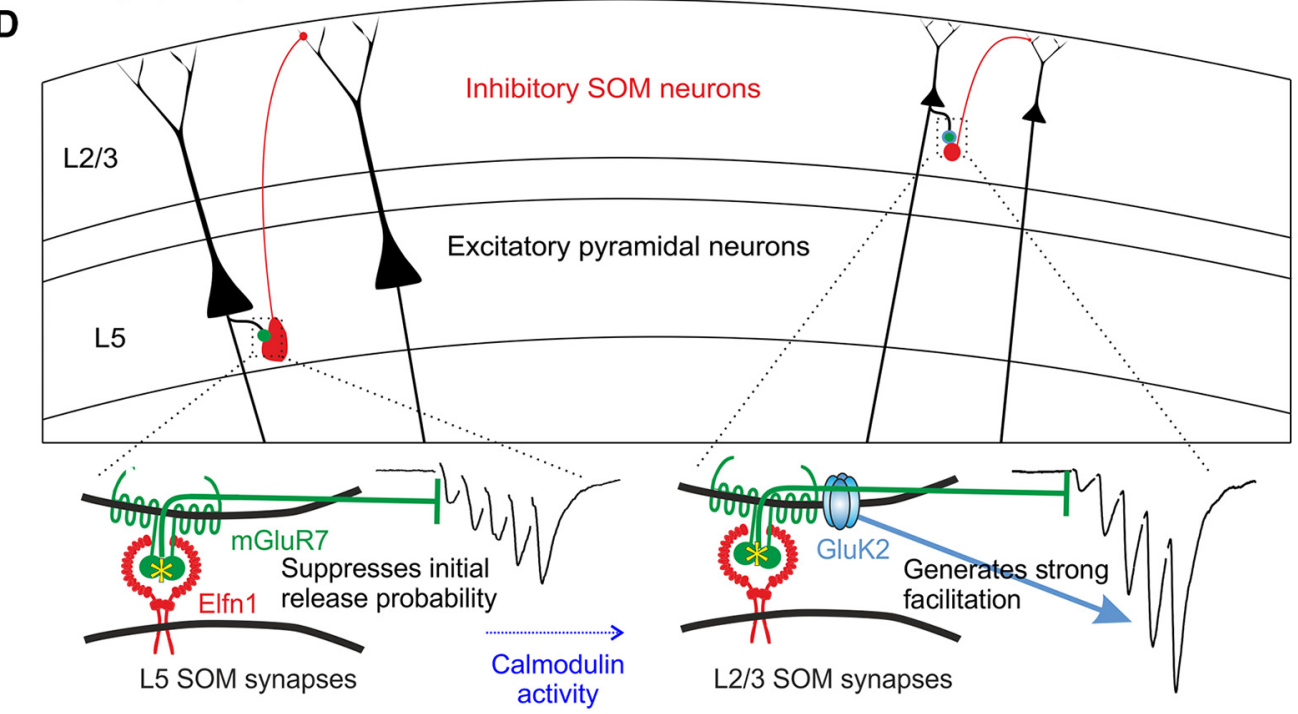

Figure 8. Calmodulin can dynamically regulate GluK2-KAR-dependent facilitation at SOM synapses. A, From HEK cells coexpressing mGluR7 and GluK2, immunoprecipitation of mGluR7 coprecipitates GluK2. The GluK2/3 immunoblot includes an input lane showing homodimers alongside the IP lane. B, From cortical tissue lysates, $\mathrm{mGluR7}$ IP also coprecipitates GluK2/3, whereas control IP with mGluR1 $\alpha$ did not. From cortical lysates of both WT and Elfn $1 \mathrm{~K} 0$ tissue, mGluR7 IP coprecipitates GluK2/3. C, Application of the calmodulin activator CALP3 (100 $\mu \mathrm{M}$ ) renders $\mathrm{L} 5 \mathrm{~S} 0 \mathrm{M}$ neurons responsive to NS102, which now suppresses facilitation onto $\mathrm{L} 5$ neurons. $\boldsymbol{D}$, Proposed mechanism underlying the layer-specific differences in the function of pyr $\rightarrow$ SOM synapses. For a train of stimuli onto L5 SOM neurons, Elfn1-mediated clustering and activation of constitutive mGluR7 generate a moderately facilitating synapse, with little to no kainate receptor activation. In L2/3 SOM neurons, early synaptic suppression with mGluR7 is followed by late synaptic facilitation with GluK2-KARs to generate a strongly facilitating synapse. Calmodulin activation can induce engagement of GluK2-KARs at L5 synapses, enabling dynamic regulation of pyr $\rightarrow$ SOM synaptic strength. Data are mean \pm SEM. ${ }^{*} p<0.05$.

labeling and the lack of specificity of the GluK2/3 antibody limit interpretability of these data. Because L5 differences could not be explained simply by absence of GluK2, we hypothesized that local protein interactions might regulate GluK2-KAR presynaptic engagement.

\section{mGluR7 acts a scaffolding structure for presynaptic GluK2-KAR recruitment}

The ubiquitous presence of mGluR7 activity at Elfn1 synapses suggested that mGluR7 might act as a protein scaffold for GluK2 recruitment. We found that mGluR7 could coimmunoprecipitate GluK2 when cotransfected into HEK cells (Fig. 8A). Likewise, mGluR7 IP from cortical lysates of both WT and Elfn1 KO animals pulled down GluK2 (Fig. 8B), which indicate that this interaction occurs, even in the absence of Elfn1. Control IP with mGluR1 $\alpha$ did not pull down GluK2. Speculating that proteinprotein interactions between mGluR7 and GluK2 at pyr $\rightarrow$ SOM synapses must somehow be regulated, we tested whether activation of calmodulin, a protein previously shown to both regulate protein interactions for the c-terminal of mGluR7 (O'Connor et al., 1999; Suh et al., 2008) and to bind GluK2 (Coussen et al., 2005), could alter GluK2 recruitment. Indeed, we discovered that activating calmodulin altered L5 $\rightarrow$ SOM synaptic responses. Calmodulin activation ( $3 \mathrm{~min}$ application of $100 \mu \mathrm{M}$ calcium like peptide 3 (CALP3), plus an additional $20 \mathrm{~min}$ for baseline stabilization) rendered synaptic facilitation NS-102-sensitive, indicating an induction of GluK2-KAR recruitment to L5 $\rightarrow$ SOM synapses (EPSC5/EPSC1, L5, CALP3 treated: $4.5 \pm 0.7$ in NS102 vs $5.0 \pm 0.9$ at baseline, $p=0.03, t=2.63$, paired $t$ test, $n / N=8 / 5$; Fig. $8 C$ ). Thus, in contrast to the Elfn1-induced constitutive synaptic suppression conveyed by mGluR7, a differential recruitment of GluK2 to L2/3 or L5 pyr $\rightarrow$ SOM synapses accounts for the observed layer-specific difference in synaptic facilitation, with calmodulin activation facilitating the engagement of GluK2 at the presynaptic terminal (Fig. $8 D$ ).

\section{Discussion}

The characteristic facilitation of pyr $\rightarrow$ SOM synapses is generated by the presence of Elfn1, which is expressed by the postsyn- 
aptic SOM neurons (Sylwestrak and Ghosh, 2012; Tomioka et al., 2014). In hippocampal SOM neurons, Elfn1-dependent synaptic facilitation is strongly influenced by synaptic frequency, where high-frequency stimulation preferentially facilitates $\mathrm{pyr} \rightarrow \mathrm{SOM}$ synapses in WT compared with Elfn1 KO animals. Strongly facilitating pyr $\rightarrow$ SOM synapses are also found in L2/3 of cortex, and this facilitation is greatly reduced in $\mathrm{KO}$ animals. At pyr $\rightarrow \mathrm{SOM}$ synapses, Elfn1 interacts with at least 2 presynaptic partners, mGluR7 and GluK2-KARs, to produce a strong short-term synaptic facilitation. Our data indicate that the mechanism of facilitation is twofold. First, the actions of mGluR7 reduce initial release probability, resulting in greater synaptic facilitation. After several consecutive action potentials, the strong facilitation in $\mathrm{L} 2 / 3$ is primarily GluK2-dependent, as application of the GluK2selective antagonist NS-102 reduces late facilitation. Notably, synapses onto L5 cortical SOM interneurons facilitate less, take longer to drive spikes, are less affected in Elfn1 KO, and are insensitive to GluK2 antagonists. Thus, the synaptic proteome dictates functional features of synaptic transmission in a synapsespecific and laminar-specific fashion.

Elfn 1 transcellular recruitment of mGluR7 clusters the receptor at HEK cell tight junction interfaces and at terminals presynaptic to SOM neurons where it generates constitutive suppression of synaptic transmission. Dimerization-induced mGluR activation is due to a conformational change in the ligand binding domain, which can be antagonized with orthosteric antagonists (Levitz et al., 2016). Elfn1-mGluR7 interactions generate a structurally induced constitutive GPCR activation, tonically suppressing pyr $\rightarrow$ SOM transmission. A previous report indicates that Elfn1 induces negative allosteric modulation of G-protein signaling in response to glutamate (Dunn et al., 2018). Although Elfn1induced negative allosteric modulation is not explicitly tested here, a reduction in L-AP4 mediated synaptic suppression for WT versus Elfn1 KO provides support for these in vitro findings. Elfn1 interactions would thus serve to bias mGluR7 activity toward a tonic signaling mode rather than a glutamate-induced signaling mode, which may help explain observations that mGluR7 are present and functional at GABAergic presynaptic terminals, where high levels of glutamate are unlikely to be found (Somogyi et al., 2003; Summa et al., 2013). In addition, we find a divergence in the synaptic outcome of allosteric modulation of the mGluR7 receptor with $30 \mathrm{nM}$ AMN082 compared with agonism with L-AP4. Allosteric modulation enhances the "delay" function of mGluR7 by further suppressing initial release, whereas agonism suppresses late release, resulting in inverse pharmacological profiles of synaptic modulation.

Within a train of high-frequency activity, synaptic suppression is transient, resulting in delayed recruitment of SOM interneurons. Thus, Elfn1 activation of mGluR7 generates a synapse that responds robustly to stimulation when it occurs at high frequency, a hallmark feature of SOM neurons in hippocampus and cortex (Pouille and Scanziani, 2004; Sylwestrak and Ghosh, 2012; Hu and Agmon, 2016). Excitatory transmission onto SOM neurons initially fails (median in vivo failure rate of unitary synaptic potentials $=80 \%$ ) but rapidly facilitates from the initial suppressed state during high-frequency spike trains (Pala and Petersen, 2015). Interestingly, this suggests that highfrequency (gamma band or high beta band) cortical activity may strongly drive the recruitment of somatostatin neuron-mediated inhibition. Indeed, gamma frequency activation of SOM interneurons in lateral septum controls food-seeking behavior (Carus-Cadavieco et al., 2017).
During a spike train, transient, early, mGluR7-dependent suppression of synaptic transmission is followed by a late, sustained GluK2-KAR-dependent facilitation. Delayed recruitment of GluK2-KAR-dependent synaptic release may be related to glutamate spillover in the synaptic cleft, as has been proposed previously (Sun et al., 2009), or may also relate to the voltage-dependent activation delay of these receptors induced by GluK2-KAR sensitivity to intracellular polyamine block (Bowie and Mayer, 1995). Delayed recruitment of presynaptic GluK2-KARs substantially contributes to synaptic facilitation at late EPSCs at pyr $\rightarrow$ SOM synapses (Sylwestrak and Ghosh, 2012), and these receptors have been proposed as spike timing dependent "conditional amplifiers" of spike transmission during natural stimulus trains at hippocampal mossy fiber synapses (Sachidhanandam et al., 2009). Presynaptic GluK2-KARs thus represent a means of enhancing SOM interneuron recruitment, as evidenced by the reduced number of stimuli required to recruitment SOM spiking for L2/3 neurons than for L5 neurons. The ability to modify GluK2-KAR engagement with the activation of calmodulin thus provides a potential mechanism for regulating the strength of SOM interneuron recruitment.

Elfn1 generates strongly facilitating synapses through the contribution of early, constitutive mGluR7 activity, and late, conditional kainate receptor recruitment, with both components contributing to the distinctive timing of pyr $\rightarrow$ SOM synapses. This stepwise construction of synaptic function provides for both a "hardwired" genetic structure and for a tunable structure to allow for both stability and flexibility in the synaptic recruitment of SOM neuron-mediated inhibition.

\section{References}

Beech DJ, Bernheim L, Hille B (1992) Pertussis toxin and voltage dependence distinguish multiple pathways modulating calcium channels of rat sympathetic neurons. Neuron 8:97-106.

Bowie D, Mayer ML (1995) Inward rectification of both AMPA and kainate subtype glutamate receptors generated by polyamine-mediated ion channel block. Neuron 15:453-462.

Cao Y, Sarria I, Fehlhaber KE, Kamasawa N, Orlandi C, James KN, Hazen JL, Gardner MR, Farzan M, Lee A, Baker S, Baldwin K, Sampath AP, Martemyanov KA (2015) Mechanism for selective synaptic wiring of rod photoreceptors into the retinal circuitry and its role in vision. Neuron 87: $1248-1260$.

Carus-Cadavieco M, Gorbati M, Ye L, Bender F, van der Veldt S, Kosse C, Börgers C, Lee SY, Ramakrishnan C, Hu Y, Denisova N, Ramm F, Volitaki E, Burdakov D, Deisseroth K, Ponomarenko A, Korotkova T (2017) Gamma oscillations organize top-down signalling to hypothalamus and enable food seeking. Nature 542:232-236.

Coussen F, Perrais D, Jaskolski F, Sachidhanandam S, Normand E, Bockaert J, Marin P, Mulle C (2005) Coassembly of two GluR6 kainate receptor splice variants within a functional protein complex. Neuron 47:555-566.

DeNardo LA, de Wit J, Otto-Hitt S, Ghosh A (2012) NGL-2 regulates inputspecific synapse development in CA1 pyramidal neurons. Neuron 76: 762-775.

de Wit J, Ghosh A (2016) Specification of synaptic connectivity by cell surface interactions. Nat Rev Neurosci 17:22-35.

Dolan J, Mitchell KJ (2013) Mutation of Elfn1 in mice causes seizures and hyperactivity. PLoS One 8:e80491.

Dunn HA, Patil DN, Cao Y, Orlandi C, Martemyanov KA (2018) Synaptic adhesion protein ELFN1 is a selective allosteric modulator of group III metabotropic glutamate receptors in trans. Proc Natl Acad Sci U S A 115:5022-5027.

Evangelista C (2004) Dose response. Computer software, MATLAB Central File Exchange. Available at: https://www.mathworks.com/matlabcentral/ fileexchange/?term = authorid\%3A11827.

Faber DS, Korn H (1991) Applicability of the coefficient of variation method for analyzing synaptic plasticity. Biophys J 60:1288-1294.

Hu H, Agmon A (2016) Differential excitation of distally versus proximally 
targeting cortical interneurons by unitary thalamocortical bursts. J Neurosci 36:6906-6916.

Hu H, Cavendish JZ, Agmon A (2013) Not all that glitters is gold: off-target recombination in the somatostatin-IRES-Cre mouse line labels a subset of fast-spiking interneurons. Front Neural Circuits 7:195.

Kammermeier PJ (2015) Constitutive activity of metabotropic glutamate receptor 7. BMC Neurosci 16:17.

Levitz J, Habrian C, Bharill S, Fu Z, Vafabakhsh R, Isacoff EY (2016) Mechanism of assembly and cooperativity of homomeric and heteromeric metabotropic glutamate receptors. Neuron 92:143-159.

Lindemann L, Jaeschke G, Michalon A, Vieira E, Honer M, Spooren W, Porter R, Hartung T, Kolczewski S, Büttelmann B, Flament C, Diener C, Fischer C, Gatti S, Prinssen EP, Parrott N, Hoffmann G, Wettstein JG (2011) CTEP: a novel, potent, long-acting, and orally bioavailable metabotropic glutamate receptor 5 inhibitor. J Pharmacol Exp Ther 339:474-486.

Losonczy A, Somogyi P, Nusser Z (2003) Reduction of excitatory postsynaptic responses by persistently active metabotropic glutamate receptors in the hippocampus. J Neurophysiol 89:1910-1919.

Mitsukawa K, Yamamoto R, Ofner S, Nozulak J, Pescott O, Lukic S, Stoehr N, Mombereau C, Kuhn R, McAllister KH, van der Putten H, Cryan JF, Flor PJ (2005) A selective metabotropic glutamate receptor 7 agonist: Activation of receptor signaling via an allosteric site modulates stress parameters in vivo. Proc Natl Acad Sci U S A 102:18712-18717.

O’Connor V, El Far O, Bofill-Cardona E, Nanoff C, Freissmuth M, Karschin A, Airas JM, Betz H, Boehm S (1999) Calmodulin dependence of presynaptic metabotropic glutamate receptor signaling. Science 286:1180-1184.

Oleskevich S, Clements J, Walmsley B (2000) Release probability modulates short-term plasticity at a rat giant terminal. J Physiol 524:513-523.

Pala A, Petersen CC (2015) In vivo measurement of cell-type-specific synaptic connectivity and synaptic transmission in layer $2 / 3$ mouse barrel cortex. Neuron 85:68-75.

Pelkey KA, McBain CJ (2008) Target-cell-dependent plasticity within the mossy fibre-CA3 circuit reveals compartmentalized regulation of presynaptic function at divergent release sites. J Physiol 586:1495-1502.

Pouille F, Scanziani M (2004) Routing of spike series by dynamic circuits in the hippocampus. Nature 429:717-723.

Reyes A, Lujan R, Rozov A, Burnashev N, Somogyi P, Sakmann B (1998) Target-cell-specific facilitation and depression in neocortical circuits. Nat Neurosci 1:279-285.

Rubio-Marrero EN, Vincelli G, Jeffries CM, Shaikh TR, Pakos IS, Ranaivoson FM, von Daake S, Demeler B, De Jaco A, Perkins G, Ellisman MH, Trewhella J, Comoletti D (2016) Structural characterization of the extracellular domain of CASPR2 and insights into its association with the novel ligand contactin1. J Biol Chem 291:5788-5802.

Sachidhanandam S, Blanchet C, Jeantet Y, Cho YH, Mulle C (2009) Kainate receptors act as conditional amplifiers of spike transmission at hippocampal mossy fiber synapses. J Neurosci 29:5000-5008.

Sansig G, Bushell TJ, Clarke VR, Rozov A, Burnashev N, Portet C, Gasparini F, Schmutz M, Klebs K, Shigemoto R, Flor PJ, Kuhn R, Knoepfel T,
Schroeder M, Hampson DR, Collett VJ, Zhang C, Duvoisin RM, Collingridge GL, van Der Putten H (2001) Increased seizure susceptibility in mice lacking metabotropic glutamate receptor 7. J Neurosci 21: $8734-8745$

Shigemoto R, Kulik A, Roberts JD, Ohishi H, Nusser Z, Kaneko T, Somogyi P (1996) Target-cell-specific concentration of a metabotropic glutamate receptor in the presynaptic active zone. Nature 381:523-525.

Somogyi P, Dalezios Y, Luján R, Roberts JD, Watanabe M, Shigemoto R (2003) High level of mGluR7 in the presynaptic active zones of select populations of GABAergic terminals innervating interneurons in the rat hippocampus. Eur J Neurosci 17:2503-2520.

Suh YH, Pelkey KA, Lavezzari G, Roche PA, Huganir RL, McBain CJ, Roche KW (2008) Corequirement of PICK1 binding and PKC phosphorylation for stable surface expression of the metabotropic glutamate receptor mGluR7. Neuron 58:736-748.

Sukoff Rizzo SJ, Leonard SK, Gilbert A, Dollings P, Smith DL, Zhang MY, Di L, Platt BJ, Neal S, Dwyer JM, Bender CN, Zhang J, Lock T, Kowal D, Kramer A, Randall A, Huselton C, Vishwanathan K, Tse SY, Butera J, et al. (2011) The metabotropic glutamate receptor 7 allosteric modulator AMN082: a monoaminergic agent in disguise? J Pharmacol Exp Ther 338:345-352.

Summa M, Di Prisco S, Grilli M, Usai C, Marchi M, Pittaluga A (2013) Presynaptic mGlu7 receptors control GABA release in mouse hippocampus. Neuropharmacology 66:215-224.

Sun HY, Dobrunz LE (2006) Presynaptic kainate receptor activation is a novel mechanism for target cell-specific short-term facilitation at Schaffer collateral synapses. J Neurosci 26:10796-10807.

Sun HY, Bartley AF, Dobrunz LE (2009) Calcium-permeable presynaptic kainate receptors involved in excitatory short-term facilitation onto somatostatin interneurons during natural stimulus patterns. J Neurophysiol 101:1043-1055.

Sylwestrak EL, Ghosh A (2012) Elfn1 regulates target-specific release probability at CA1-interneuron synapses. Science 338:536-540.

Tan Z, Hu H, Huang ZJ, Agmon A (2008) Robust but delayed thalamocortical activation of dendritic-targeting inhibitory interneurons. Proc Natl Acad Sci U S A 105:2187-2192.

Tomioka NH, Yasuda H, Miyamoto H, Hatayama M, Morimura N, Matsumoto Y, Suzuki T, Odagawa M, Odaka YS, Iwayama Y, Won Um J, Ko J, Inoue Y, Kaneko S, Hirose S, Yamada K, Yoshikawa T, Yamakawa K, Aruga J (2014) Elfn1 recruits presynaptic mGluR7 in trans and its loss results in seizures. Nat Commun 5:4501.

Ullmer C, Zoffmann S, Bohrmann B, Matile H, Lindemann L, Flor P, Malherbe $P$ (2012) Functional monoclonal antibody acts as a biased agonist by inducing internalization of metabotropic glutamate receptor 7 . Br J Pharmacol 167:1448-1466.

Williams ME, Wilke SA, Daggett A, Davis E, Otto S, Ravi D, Ripley B, Bushong EA, Ellisman MH, Klein G, Ghosh A (2011) Cadherin-9 regulates synapse-specific differentiation in the developing hippocampus. Neuron 71:640-655. 